\title{
Metamorphism of basement rocks in the Central Zone of the North China Craton: implications for Paleoproterozoic tectonic evolution
}

\author{
Guochun Zhao a,b,*, Peter A. Cawood ${ }^{\mathrm{a}}$, Simon A. Wilde ${ }^{\mathrm{a}}$, Min Sun ${ }^{\mathrm{b}}$, \\ Liangzhao $\mathrm{Lu}^{\mathrm{c}}$ \\ a Tectonics Special Research Centre, Curtin University of Technology, School of Applied Geology, GPO Box U1987, Perth, \\ WA 6845, Australia \\ b Department of Earth Sciences, The University of Hong Kong, Pokfulam Road, Hong Kong \\ ${ }^{\mathrm{c}}$ College of Earth Sciences, Changchun University of Science \& Technology, Changchun 130026, People's Republic of China
}

Received 11 August 1998; accepted 24 March 2000

\begin{abstract}
Lithological, structural, metamorphic and geochronological data for the North China Craton enable its division into the Western and Eastern Blocks of Archean to Paleoproterozoic age separated by a north-south trending Paleoproterozoic orogenic belt: the Central Zone. The Central Zone is divisible into a series of low- to medium-grade granite-greenstone belts and high-grade metamorphic terrains containing reworked Archean material and late Archean to Paleoproterozoic juvenile igneous and sedimentary rocks which developed in intra-continental magmatic arc and intra-arc basin environments bordering the western margin of the Eastern Block. The basement rocks from the Central Zone, regardless of their protolith age, composition and metamorphic grade, record a metamorphic history characterized by nearly isothermal decompression $\left(\mathrm{M}_{2}\right)$ and then retrogressive cooling $\left(\mathrm{M}_{3}\right)$ following peak metamorphism $\left(\mathrm{M}_{1}\right)$. The decompression textures are represented by worm-like hypersthene + plagioclase symplectites or clinopyroxene + orthopyroxene + plagioclase coronas in mafic granulites, hornblende/cummingtonite + plagioclase symplectites in amphibolites, and cordierite coronas and cordierite + orthopyroxene or cordierite + spinel symplectites in pelitic rocks. The cooling textures are shown by hornblende + plagioclase symplectites in mafic granulites, chlorite + epidote + mica retrogressive rims around garnet or hornblende grains in amphibolites, and biotite $+\mathrm{K}-$ feldspar \pm muscovite \pm magnetite replacing garnet, cordierite and sillimanite in pelitic gneisses. These textural relations and their $\mathrm{P}-\mathrm{T}$ estimates define near-isothermal decompressional clockwise $\mathrm{P}-\mathrm{T}$ paths, which, in combination with lithological, structural and geochronological constraints, are in accord with collision between the Eastern and Western Blocks of the North China Craton at 1.8 Ga. (C) 2000 Elsevier Science B.V. All rights reserved.
\end{abstract}

Keywords: Continental collision; Metamorphism; North China Craton; P-T paths; Tectonothermal evolution

* Corresponding author. Tel.: + 61-8-92663421; fax: + 61-8-92663153.

E-mail address: zhao@fithos.curtin.edu.au (G. Zhao). 


\section{Introduction}

The variations of pressure and temperature that characterize a metamorphic event are a function of the tectonic setting and of the processes that were active during metamorphism (England and Thompson, 1984; Thompson and England, 1984; Bohlen, 1987; Harley, 1989; Brown, 1993). Thus, the thermal evolution of a metamorphic belt has been regarded as one of the most important keys to understanding the tectonic history of complex metamorphic terranes, and metamorphic $\mathrm{P}-\mathrm{T}$ paths have been used successfully to constrain the tectonic setting and processes (Harley, 1985, 1988; Mezger et al., 1990; Bohlen, 1991; Mengel and Rivers, 1991). Generally, clockwise, especially isothermal decompressional, $\mathrm{P}-\mathrm{T}$ paths are considered to develop in continental collisional environments (England and Thompson, 1984; Thompson and England, 1984; Bohlen, 1991; Brown, 1993), whereas anticlockwise, especially isobaric cooling, $\mathrm{P}-\mathrm{T}$ paths are interpreted to be related to the intrusion and underplating of mantle-derived magma which may occur in intracontinental magmatic arc regions (Wells, 1980; Bohlen, 1987, 1991), hot spots related to mantle plumes (Bohlen, 1991) and incipient rift environments (Sandiford and Powell, 1986). However, inferences about tectonic setting and process based on $\mathrm{P}-\mathrm{T}$ paths are often complicated by variations in $\mathrm{P}-\mathrm{T}$ data from different rock units or tectonic domains within the same terrane (Selverstone and Chamberlain, 1990). For example, contrasting $\mathrm{P}-\mathrm{T}$ paths have been observed between anticlines and adjacent synclines, or between hanging and foot wall of the same fault (Chamberlain and Karabinos, 1987).

As one of the best exposed Archean to Paleoproterozoic cratonic blocks in the world, the North China Craton is a promising area for applying the large-scale synthesis approach of metamorphic $\mathrm{P}-\mathrm{T}$ paths to understanding tectonic settings and processes, since numerous studies on the tectonothermal evolution of basement rocks have been undertaken throughout the craton in the past decade and a $\mathrm{P}-\mathrm{T}$ data base, largely published in Chinese, is now available (Cui et al., 1991; Jin et al., 1991; Lu, 1991; Li, 1993; Liu et al., 1993; Lu and Jin, 1993; Sun et al., 1993a; Chen et al., 1994; Ge et al., 1994; Zhao et al., 1998, 1999a). Zhao et al. (1998, 1999a) have summarized the $\mathrm{P}-\mathrm{T}$ paths of basement rocks in the eastern and western parts of the craton and discussed their tectonic implications. On the basis of lithological, structural, metamorphic and geochronological data, Zhao et al. (1998, 1999a) proposed that the North China Craton may have been composed of two separate continental blocks, called the Eastern and Western Blocks, from the late Archean to Paleoproterozoic and the $\sim 1.8 \mathrm{Ga}$ collision between these two blocks along the Central Zone resulted in the final amalgamation of the North China Craton (Wu et al., 1991; Wu and Zhong, 1998; Zhao et al., 1998, 1999a). The purpose in this communication is to examine the collision-related tectonothermal evolution of various rock units and metamorphic domains in the Central Zone and, in combination with lithological, structural and geochronological data to further constrain the Paleoproterozoic amalgamation of the Eastern and Western Blocks which formed the North China Craton.

\section{Regional setting}

The North China Craton is the largest and oldest known cratonic block in China, covering an area of more than $1500000 \mathrm{~km}^{2}$, and is bounded by faults and younger orogenic belts (Fig. 1). The early Paleozoic Qilianshan (QLS) Orogen and the late Paleozoic Tianshan-Inner Mongolia-Daxinganling (TIMD) Orogen bound the craton to the west and the north, respectively, whereas in the south the Mesozoic Qinling-Dabie-Su-Lu (QDSL) high- to ultrahigh-pressure belt separates the craton from the South China Craton (Fig. 1).

The tectonic evolution of the North China Craton is poorly constrained. Traditionally, it has been considered to be composed of a uniform Archean crystalline basement, overlain by a Proterozoic to Cainozoic cover, and its history was explained using a pre-plate tectonic geosynclinal-style model (Huang, 1977). Terrane accretion and collisional models have only been 
applied recently (Li et al., 1990; Zhai et al., 1992; Wang et al., 1996; Zhao et al., 1999a,b), including recognition of a Paleoproterozoic orogen along the central North China Craton, named the Central Zone, which separates the craton into Western and Eastern Blocks (Fig. 2; Zhao et al., 1999a,c).

The Eastern Block includes the MiyunChengde, Eastern Hebei, Western Liaoning, Western Shangdong, Eastern Shandong, Southern Liaoning, Northern Liaoning and Southern Jilin domains (Fig. 2). It consists predominantly of Late Archean domiform tonalitic-trondhjemiticgranodioritic (TTG) batholiths and -2.5 Ga syntectonic granites outlined by anastomosing networks and linear belts of open to tight synforms of minor volcanic and sedimentary rocks metamorphosed in greenschist to granulite facies at $\sim 2.5 \mathrm{Ga}$, with anticlockwise $\mathrm{P}-\mathrm{T}$ paths (Cui et al., 1991; Li, 1993; Ge et al., 1994; Sun et al., 1993b; Song et al., 1996; Kröner et al., 1998; Zhao et al., 1998). Some Early to Middle Archean rocks are locally present in the Eastern Block (Huang et al., 1986; Jahn et al., 1987; Qiao et al., 1987; Liu et al., 1992; Song et al., 1996), but their tectonic nature and deformational and metamorphic history are unclear due to reworking during the $\sim 2.5 \mathrm{Ga}$ tectonothermal event. The Western Block, including the Helanshan-Qianlishan, Daqingshan-Ulashan, Guyang-Wuchuan, Sheerteng and Jining domains (Fig. 2), has a late Archean lithological assemblage, structural style and metamorphic history similar to that of the Eastern Block (Li et al., 1987; Shen et al., 1987, 1990; Jin et al., 1991; Liu et al., 1993; Lu and Jin, 1993), but differs by the absence of Early to Middle Archean assemblages and by being overlain by and interleaved with Paleoproterozoic khondalites. The latter, defined as a suite of granulite facies supracrustal rocks including graphitebearing sillimanite-garnet gneisses and associated garnet-bearing felsic paragneisses (leptynites), quartzites, calc-silicate rocks and marbles, were affected by a $\sim 1.8 \mathrm{Ga}$ metamorphic event char-

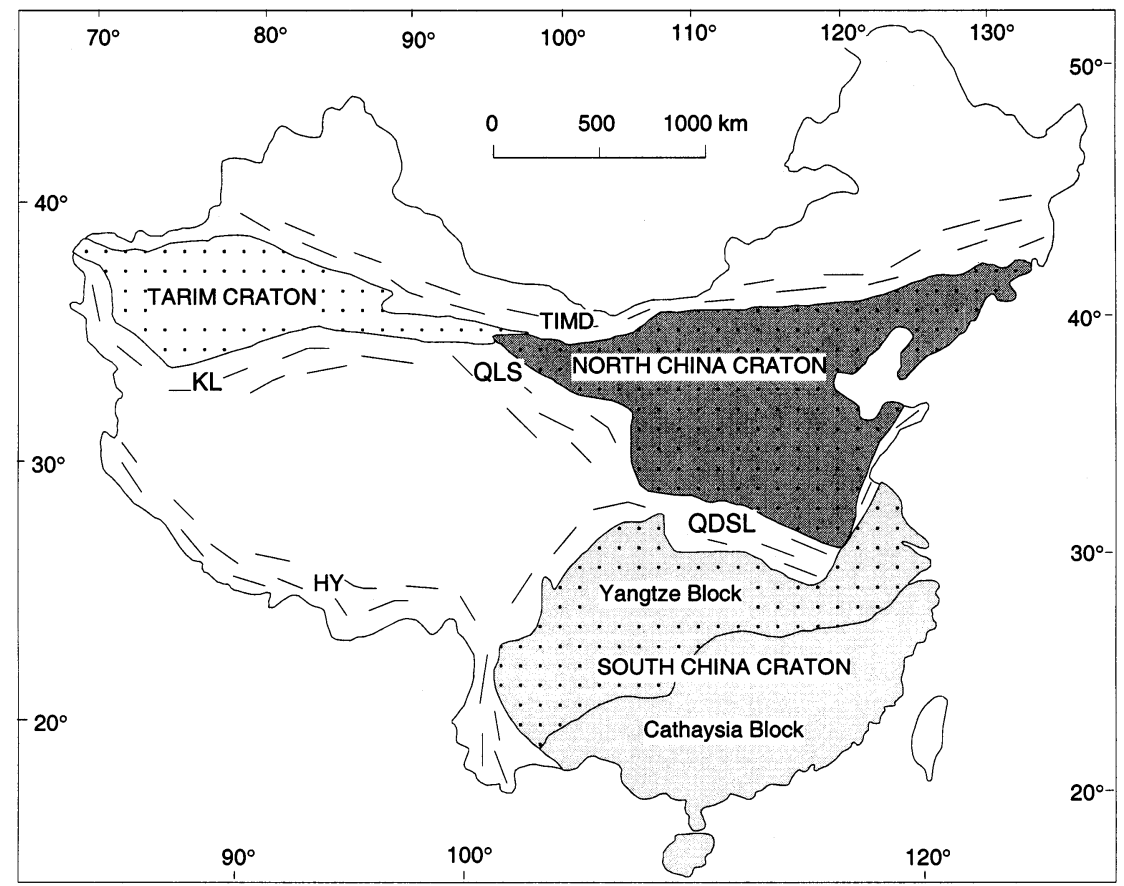

Fig. 1. Outline tectonic map of China showing the major Precambrian blocks and the Late Neoproterozoic and Paleozoic: fold belts. HY, Himalaya fold belt; KL, Kunlun fold belt; QDSL, Qinling-Dabie-Su-Lu fold belt; QLS, Qilianshan fold belt; TIMD, Tianshan-Inner Mongolia-Daxinganling fold belt. 


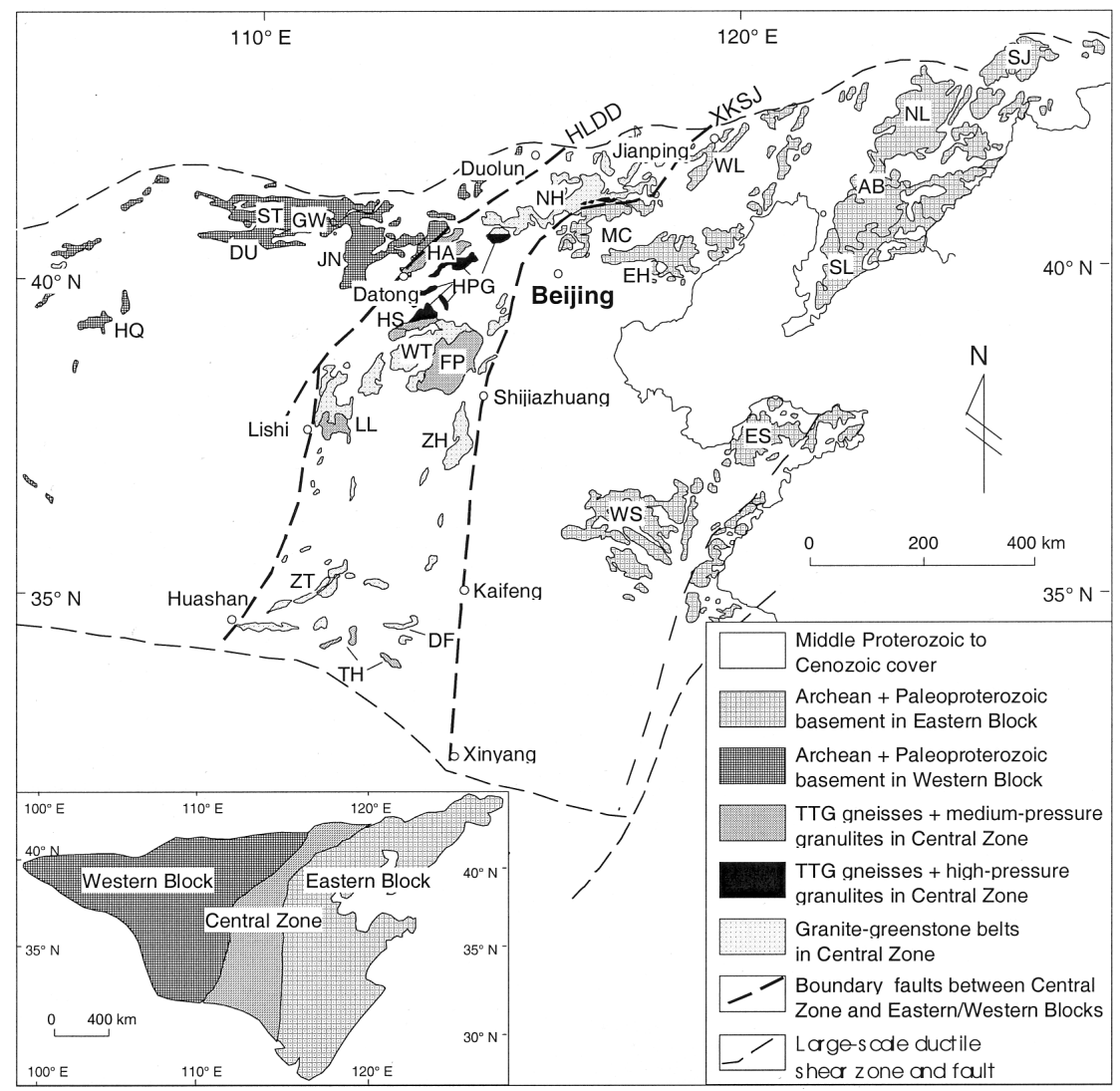

Fig. 2. Distribution of the basement rocks in the North China Craton and the distribution of the Eastern and Western Blocks and the Central Zone (Inset). The Eastern Block includes the Anshan-Benxi (AB), Eastern Hebei (EH), Eastern Shandong (ES), Myun-Chengde (MC), Northern Liaoning (NL), Southern Jilin (SJ), Southern Liaoning (SL), Western Liaoning (WL) and Western Shandong (WS) domains; Western Block includes the Daqingshan-Ulashan (DU), Guyang-Wuchuan (GW), Helanshan-Qianlishan (HQ), Jinning (JN) and Sheerteng (ST) domains; and the Central Zone includes the Dengfeng (DF), Fuping (FP), Hengshan (HS), High-Pressure Granulite (HPG), Huaian (HA), Lüliang (LL), Northern Hebei (NH), Taihua (TH), Wutai (WT), Zanhuang $(\mathrm{ZH})$ and Zhongtiao (ZT) domains. HLDD, Huashan-Lishi-Datong-Duolun Fault; XKSJ, Xinyang-Kaifeng-Shijiazhuang-Jianping Fault.

acterized by clockwise $\mathrm{P}-\mathrm{T}$ paths (Jin et al., 1991; Lu, 1991; Liu et al., 1993; Lu and Jin, 1993; Zhao et al., 1999a).

Separating the two blocks is the Central Zone which extends as a north-south trending belt across the North China Craton (Fig. 2). The zone consists of a series of greenschist- to granulite-facies metamorphic terrains containing re-worked Archean components derived from the Western and Eastern Blocks, together with late Archean to Paleoproterozoic juvenile igneous and sedimentary rocks. Geochemical studies of metamorphosed mafic rocks suggest that the basement rocks in the
Central Zone developed in continental magmatic are and intra-arc basin environments (Bai, 1986; Geng and Wu, 1990; Wu et al., 1991; Zhang et al., 1991; Bai et al., 1992; Wang et al., 1996) and were metamorphosed in greenschist to granulite facies at about 1.9-1.8 Ga (Wilde et al., 1997, 1998; Wu et al., 1997; Zhao et al., 1999b; Zhao et al., in press). These arcs and intra-arc basins most likely developed on the western margin of the Eastern Block, since most reworked mid-Archean TTG gneisses and granulites discovered in the Central Zone show compositional features and isotopic ages similar to those in the Eastern Block (Zhao et al., 1999c). 
The Central Zone is separated from the Western Block by the Huashan-Lishi-Datong-Duolun (HLDD) Fault and from the Eastern Block by the Xingyang-Kaifeng-Shijiazhuang-Jiianping (XKSJ) Fault (Fig. 2). Both faults strike $\mathrm{N}-\mathrm{S}$ in the central and southern parts and turn to $\mathrm{N}-\mathrm{E}$ in the north (Fig. 2). The central and southern segments of the XKSJ Fault are also called the Zhuoxian-Shijiazhuang Fault and XingtaiAnyang Fault and constitute part of a major fault system in the eastern part of China (Ren, 1980). The presence of voluminous mantle-derived basalts exposed along the two faults suggests that these faults are deep-seated, possibly reaching into the lower crust or upper mantle (Ren, 1980). Geophysical data indicate a westward increase of depth to the Moho across the Central Zone from $37 \mathrm{~km}$ in the east along the XKSJ Fault to $42 \mathrm{~km}$ in the west along the HLDD Fault (Ren, 1980). The exact ages of these two faults have not been determined, but a major phase of activity was presumably in the Mesozoic, as shown by the eruption of voluminous basalts along the faults (Ren, 1980). Therefore, whether these two faults represent the original fundamental boundaries between the two blocks and the Central Zone remains unknown. The interpretation preferred here is that the two faults represent cryptic Late Archean to Paleoproterozoic tectonic boundaries that were reactivated during the Mesozoic.

\section{Basement rocks in the Central Zone and their radiometric ages}

The Central Zone of the North China Craton consists of the Dengfeng, Fuping, Hengshan, Huaian, Lüliang, northern Hebei, Taihua, Wutai, Zanhuang, Zhongtiao and high-pressure granulite (HPG) domains, with boundaries defined by faults or obscured by younger rock units (Fig. 2). These domains consist predominantly of varying proportions of late Archean to Paleoproterozoic basement rocks metamorphosed in greenschist to granulite facies. On the basis of lithology and metamorphic grade, the basement rocks in the Central Zone can be further divided into highgrade areas and low-grade granite-greenstone belts. The former include the Fuping, Hengshan, Huaian, HPG and Taihua domains and are composed predominantly of late Archean TTG gneisses and supracrustal rocks with minor amounts of Paleoproterozoic: upper amphibolite to granulite facies metavolcanics and metasediments. The granite-greenstone belts include the Dengfeng, Lüliang, Wutai, Zhongtiao and Zanhuang domains and are dominated by late Archean to Paleoproterozoic granites and associated greenstone sequences metamorphosed in lower greenschist to amphibolite facies. The northern Hebei domain contains a number of small-scale, high-grade granulite facies belts surrounded by low-grade granite-greenstone belts. In most cases, basement rocks in the low-grade granite-greenstone belts show a continuation from the late Archean to Paleoproterozoic. The largest outcrop of such a segment is in the Wutai (including the Hutuo Group) domain that comprises the Lower Wutai Group (2.55 Ga), the Upper Wutai Group (2.10 Ga), and the Hutuo Group dated at 1.90-1.80 Ga (Liu et al., 1985; Tian, 1991; Bai and Dai, 1998; Cawood et al., 1998; Wilde et al., 1998).

Radiometric dating efforts have been concentrated mostly in the Wutai, Zhongtiao, HPG, Huaian, Fuping, Lüliang, Dengfeng domains, whereas age data from the Hengshan and Taihua domains are very sparse and no ages have been reported from the northern Hebei and Zanhuang domains. The available radiometric ages are shown schematically in Fig. 3. With the present scarcity of data it is unknown whether there is the early Archean $(>3.2 \mathrm{Ga})$ crust in the Central Zone of the North China Craton, although a $3323 \pm 44 \mathrm{U}-\mathrm{Pb}$ single zircon age has been reported from 'old' tonalitic gneiss from the Huaian domain (Kröner et al., 1987). A few ages of $\sim 2.8$ Ga have been reported from TTG gneisses, mafic granulites and amphibolites in the Huaian, Hengshan, Fuping, Taihua and Dengfen domains (Fig. 3; Liu et al., 1985; Kröner et al., 1988; Tian et al., 1992; Wang et al., 1995). These ages are insufficient to document the existence of mid-Archean crust in the Central Zone and most likely represent minor amounts of reworked mid-Archean components derived from the Eastern and/or 
Western Blocks. It appears from the present data that much of the basement framework of the Central Zone was established after $2.6 \mathrm{Ga}$, with a major crust-forming event at $\sim 2.5 \mathrm{Ga}$ (Fig. 3). This included mafic volcanism and TTG magmatism in the high grade areas, such as the Huaian,
Hengshan, Fuping, Taihua domains, and the eruption of basalts, dacites, rhyodacites and andesites and emplacement of granites in the granitegreenstone belts, such as the Wutai, Lüliang, Zhongtiao and Dengfen domains (Fig. 3; Chen et al., 1980; Liu et al., 1985; Kröner et al., 1988;

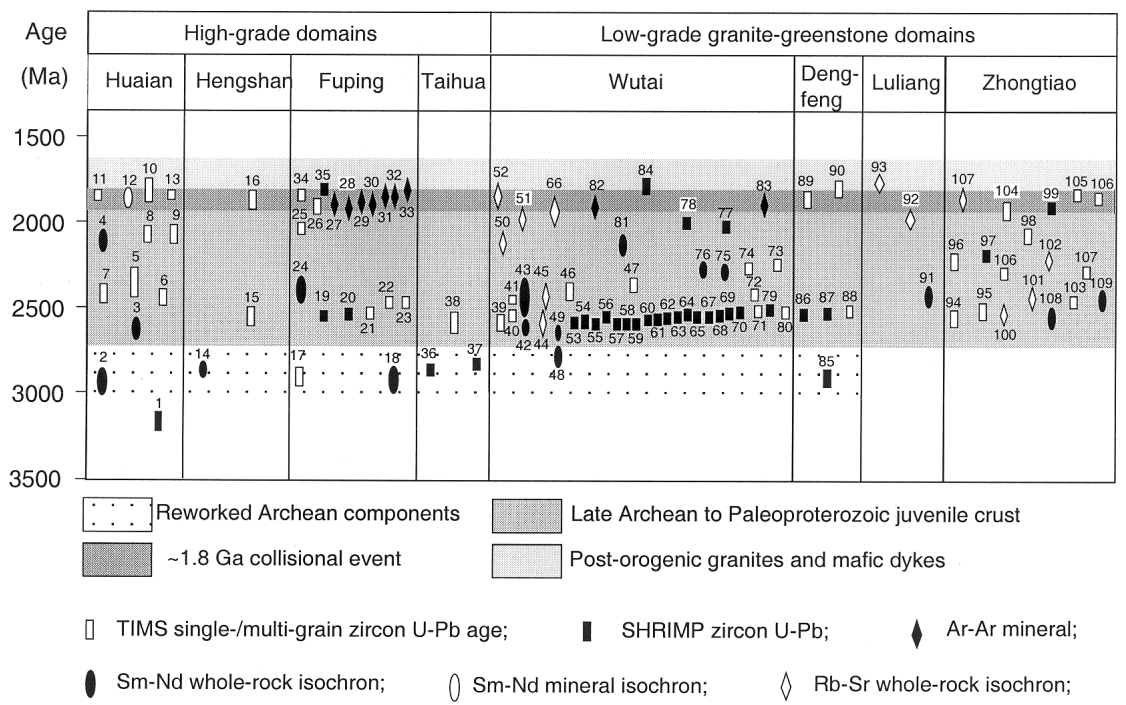

Fig. 3. Available radiometric data from the Central Zone of the North China Craton. Except where noted, all ages are $\mathrm{U}-\mathrm{Pb}$ zircon ages: 1, 'old' tonalitic gneiss (Kröner et al., 1987); 2, medium-pressure granulites, Sm-Nd whole-rock isochron (Wang et al., 1995); 3, high-pressure granulites, Sm-Nd whole-rock isochron (Shen et al., 1994; Guo et al., 1996); 4, high-pressure metamorphosed dykes, Sm-Nd whole-rock isochron (Guo et al., 1996); 5, medium-pressure granulites (Wang et al., 1995); 6, TTG gneisses (Shen et al., 1990; Wang et al., 1995); 7, high-pressure granulites (Shen et al., 1994); 8, potassium granite (Guo et al., 1996); 9, mafic dyke (Wang et al., 1995); 10, high-pressure granulites (Guo et al., 1996); 11, pelitic gneiss (Guo et al., 1996); 12, garnet-pyroxene-hornblende Sm-Nd isochron age of high-pressure granulites (Guo et al., 1996); 13, charnockite (Guo et al., 1996); 14, medium-pressure granulites, $\mathrm{Sm}-\mathrm{Nd}$ whole-rock isochron (Tian et al., 1992); 15, lower intercept of multi-grain zircon population from tonalitic gneiss (Tian et al., 1992); 16, upper intercept of multi-grain zircon population from tonalitic gneiss (Tian et al., 1992); 17, 'old' tonalitic gneiss (Liu et al., 1985); 18, amphibolite, Sm-Nd whole-rock isochron (Zhang et al., 1991); 19-20, tonalitic gneiss and granitic gneiss (Wilde, SA, unpublished data); 21-22, granitic gneiss and tonalitic gneiss (Xu et al., 1995); 23, paragneiss (Liu et al., 1985); 24, amphibolite, Sm-Nd whole-rock isochron (Sun et al., 1992); 25, felsic gneiss (Liu et al., 1985); 26, granitic gneiss (Zhang et al., 1991); 27-33, granitic gneiss, biotite ${ }^{40} \mathrm{Ar} /{ }^{39} \mathrm{Ar}$ (Xu et al., 1995); 34, medium-pressure granulites (Liu et al., 1985); 35, granitic pegmatite (Wilde, SA, unpublished data); 36-37, TTG gneisses (Kröner et al., 1988); 38, TTG gneisses (Chen et al., 1980); 39-41, granites (Bai et al., 1992); 42-43, amphibolites, Sm-Nd whole-rock isochron (Wang et al., 1997); 44-45, amphibolites, $\mathrm{Rh}-\mathrm{Sr}$ whole-rock isochron (Wang et al., 1997); 46-47, amphibolite (Bai et al., 1992); 48-49, phyllite, Sm-Nd whole-rock isochron (Wang et al., 1997); 50-52, phyllite, Rb-Sr whole-rock isochron (Bai et al., 1992); 53-56, granites (Liu et al., 1985); 57-65, granites (Wilde et al., 1997); 66, syn-tectonic granite, $\mathrm{Rb}-\mathrm{Sr}$ whole-rock isochron (Sun et al., 1992); 67-70, meta-andacite/dacite (Wilde et al., 1997, 1998); 71-74, metabasalts (Bai et al., 1992; Wang et al., 1997); 75-76, metabasalts, Sm-Nd whole-rock isochron (Wang et al., 1997); 77-78, porphyritic granites (Wilde, SA, unpublished data); 79, greenschist (Wilde et al., 1997); 80, paragneiss (Liu et al., 1985); 81, metabasalt, Sm-Nd whole-rock isochron (Sun et al., 1992); 82-83, hornblende, ${ }^{40} \mathrm{Ar} /{ }^{39} \mathrm{Ar}$ (Wang et al., 1997b); 84, post-orogenic granite (Wang et al., 1997b); 85-87, meta-rhyodacite (Kröner et al., 1988); 88, metavolcanics (Chen et al., 1980); 89-90, paragneiss, (Zhang et al., 1985); 91, metamorphosed mafic volcanics, Sm-Nd whole-rock isochron (Zhang, 1988); 92, metamorphosed mafic volcanics, $\mathrm{Rb}$-Sr whole-rock isochron (Zhang, 1988); 93, metavolcanics, $\mathrm{Rb}$-Sr whole rock isochron (Qiao, 1985); 94-99, metatuff (Sun et al., 1993a); 100-102, metavolcanics, Rb-Sr whole-rock isochron (Sun et al., 1993b); 103-105, metavolcanics (Sun et al., 1993a); 106-106, granite (Sun et al., 1993b); 107, pelitic schist-marble, Rb-Sr whole-rock isochron (Sun et al., 1993a); 108-109, amphibolite, Sm-Nd whole-rock isochron (Sun et al., 1993b). 
Zhang, 1988; Bai et al., 1992; Sun et al., 1992, 1993a; Tian et al., 1992; Shen et al., 1994; Wang et al., 1995; Guo et al., 1996; Wang et al., 1997a; Wilde et al., 1997, 1998). In most domains, the eruption of mafic to felsic volcanic rocks and emplacement of TTG and granite plutons occurred during the period from 2.5 to $1.9 \mathrm{Ga}$, as recorded in $\sim 2.5 \mathrm{Ga}$ TTG gneisses and $\sim 2.3 \mathrm{Ga}$ mafic granulites and amphibolites in the Huaian and Fuping domains and $\sim 2.3 \mathrm{Ga}$ metabasalts, $\sim 2.1 \mathrm{Ga}$ porphyritic granites, $\sim 2.0 \mathrm{Ga}$ metatuffs, $\sim 1.9$ Ga mafic dykes in the Wutai, Lüliang and Zhongtiao domains (Zhang, 1988; Sun et al., 1992, 1993b; Wang et al., 1995, 1997b; Wilde et al., 1997).

The major metamorphic event affecting basement rocks of the Central Zone occurred at $\sim 1.8$ Ga. In the Fuping, Hengshan and Huaian domains, multi-grain zircon populations from TTG gneisses gave upper intercept $\mathrm{U}-\mathrm{Pb}$ ages of $\sim 2.5$ $\mathrm{Ga}$ and lower intercepts of $\sim 1.8 \mathrm{Ga}$; the latter is considered to represent the age of the tectonothermal event (Liu et al., 1985; Tian et al., 1992; Wang et al., 1995). The $1.8 \mathrm{Ga}$ tectonothermal event was also revealed by $\mathrm{Sm}-\mathrm{Nd}$ ages of garnets from the high-pressure granulites and ${ }^{40} \mathrm{Ar} /$ ${ }^{39} \mathrm{Ar}$ ages of homblendes in amphibolites and biotites in TTG gneisses (Fig. 3; Xu et al., 1995; Guo et al., 1996; Wang et al., 1997a,b). In addition, recent SHRIMP $\mathrm{U}-\mathrm{Pb}$ zircon dating has recognized two zircon age groups within TTG gneisses and supracrustal rocks from the Hengshan, Wutai and Fuping domains at $\sim 2500$ and $\sim 1800$ Ma (Wilde et al., 1997, 1998; Kröner, personal communication, 1999), where the younger ages are obtained mainly from rims of zircons and thus interpreted to date the metamorphic event.

\section{Determinations of $P-T$ paths}

Several techniques, including microstructural analysis, mineral zoning patterns, data from fluidinclusion studies and $\mathrm{P}-\mathrm{T}$ determinations made with internally consistent thermodynamic datasets have been applied to deducing metamorphic P-T paths (Zhang, 1990; Zhai et al., 1992; Mei,
1994; Liu, 1995, 1996a; Zhao et al., 1998, 1999a,b; Zhao et al., in press). In this section, we use textural criteria to infer metamorphic reactions and their relative timing, and conventional geothermobarometry, the programs THERMOCALC (Powell and Holland, 1994; Holland and Powell, 1998) and TWQ (Berman, 1991) to derive $\mathrm{P}-\mathrm{T}$ conditions from mineral compositional data. Most minerals from mafic granulites were analysed with a Link EDS system connected to a Jeol 6400 electron microprobe at the University of Western Australia, using Link's software for ZAF correction and data processing. Analyses were performed with $15 \mathrm{kV}$ accelerating voltage, $\sim 5$ nA beam current and counting time of 30-40 s. Natural and synthetic minerals were used as standards. Compositions of minerals from amphibolites were determined with an EPM-810Q electron microprobe at Beijing University, with an accelerating voltage of $\sim 15 \mathrm{kV}$, a beam current of $15-20 \mathrm{nA}$ and a spot size of 2-10 g. Natural minerals were used as standards for major elements with synthetic oxides and silicate minerals used for some minor elements. A representative selection of the minerals used for $\mathrm{P}-\mathrm{T}$ calculations is included in Tables $1-3$, and the whole dataset used for $\mathrm{P}-\mathrm{T}$ calculations in the Hengshan, Wutai, Fuping, Lüliang and HPG domains are available from the first author.

The following discussions will mainly focus on the Hengshan (HS), Wutai (WT), Fuping (FP), Lüliang (LL), Zhongtiao (ZT), Huaian (HA), HPG and Northern Hebei (NH) domains from which mineral assemblages, reaction relations, thermobarometric data and $\mathrm{P}-\mathrm{T}$ paths have been relatively well documented, whereas metamorphic and $\mathrm{P}-\mathrm{T}$ data from the Zanhuang $(\mathrm{ZH})$, Dengfeng (DF) and Taihua (TH) domains are unavailable at present.

\subsection{Hengshan domain (HS)}

The Hengshan domain is located in the central area of the Central Zone, approximately $300 \mathrm{~km}$ west-southwest of Beijing (Fig. 2). The domain is dominated by grey gneisses (TTG), with minor amounts of mafic granulites which are restricted to boudins and sheets within the grey gneisses. 
Table 1

Representative analyses of medium-pressure granulites

\begin{tabular}{|c|c|c|c|c|c|c|c|c|c|c|c|c|}
\hline \multirow[b]{2}{*}{ Sample } & \multicolumn{12}{|l|}{ Garnet } \\
\hline & $\mathrm{B} 13$ & B13 & $\mathrm{C} 75$ & $\mathrm{C} 75$ & $\mathrm{C} 13$ & $\mathrm{C} 13$ & 7518 & 7523 & 7517 & 7504 & 7527 & 7528 \\
\hline Texture $^{\mathrm{a}}$ & $\mathrm{pc}$ & $\mathrm{pr}$ & $\mathrm{pc}$ & $\mathrm{pr}$ & $\mathrm{pc}$ & $\mathrm{pr}$ & rim1 & riml & rim1 & rim2 & rim2 & rim2 \\
\hline $\mathrm{SiO}_{2}$ & 38.22 & 38.34 & 38.15 & 37.93 & 38.27 & 38.02 & 38.20 & 38.02 & 38.15 & 37.88 & 37.90 & 38.03 \\
\hline $\mathrm{TiO}_{2}$ & 0.12 & 0.00 & 0.02 & 0.06 & 0.00 & 0.05 & 0.00 & 0.08 & 0.06 & 0.07 & 0.00 & 0.00 \\
\hline $\mathrm{Al}_{2} \mathrm{O}_{3}$ & 20.98 & 20.88 & 20.95 & 20.99 & 20.89 & 21.21 & 21.11 & 20.73 & 21.03 & 21.04 & 20.88 & 21.18 \\
\hline $\mathrm{Cr}_{2} \mathrm{O}_{3}$ & 0.09 & 0.19 & 0.04 & 0.23 & 0.01 & 0.09 & 0.20 & 0.05 & 0.19 & 0.09 & 0.08 & 0.01 \\
\hline $\mathrm{FeO}$ & 27.88 & 30.06 & 25.65 & 27.99 & 25.51 & 30.06 & 27.08 & 28.13 & 28.15 & 28.88 & 28.63 & 29.44 \\
\hline $\mathrm{MnO}$ & 0.68 & 1.07 & 0.84 & 1.28 & 0.46 & 0.87 & 1.68 & 1.83 & 2.04 & 2.44 & 2.34 & 1.89 \\
\hline $\mathrm{MgO}$ & 3.07 & 3.67 & 3.85 & 3.70 & 3.02 & 3.51 & 3.49 & 3.66 & 3.67 & 3.12 & 3.42 & 3.69 \\
\hline $\mathrm{CaO}$ & 9.51 & 7.12 & 10.71 & 7.90 & 11.81 & 6.78 & 8.86 & 7.73 & 7.20 & 6.88 & 6.92 & 5.97 \\
\hline Total & 100.55 & 101.33 & 100.21 & 100.08 & 99.97 & 100.59 & 100.62 & 100.23 & 100.54 & 101.40 & 100.17 & 100.21 \\
\hline An & 0.599 & 0.633 & 0.530 & 0.603 & 0.533 & 0.651 & 0.578 & 0.599 & 0.609 & 0.628 & 0.617 & 0.645 \\
\hline Sps & 0.015 & 0.024 & 0.019 & 0.029 & 0.011 & 0.019 & 0.037 & 0.041 & 0.045 & 0.055 & 0.052 & 0.042 \\
\hline Prp & 0.120 & 0.143 & 0.150 & 0.145 & 0.120 & 0.138 & 0.136 & 0.143 & 0.144 & 0.123 & 0.135 & 0.145 \\
\hline Grs & 0.262 & 0.188 & 0.285 & 0.217 & 0.319 & 0.186 & 0.241 & 0.208 & 0.198 & 0.190 & 0.189 & 0.167 \\
\hline \multirow[t]{2}{*}{ Adr } & 0.014 & 0.037 & 0.048 & 0.020 & 0.050 & 0.017 & 0.022 & 0.029 & 0.014 & 0.012 & 0.022 & 0.004 \\
\hline & \multicolumn{12}{|c|}{ Plagioclase } \\
\hline Sample & B13 & B13 & $\mathrm{C} 75$ & $\mathrm{C} 75$ & $\mathrm{C} 13$ & $\mathrm{C} 13$ & 7518 & 7523 & 7517 & 7504 & 7527 & 7528 \\
\hline Texture $^{\mathrm{a}}$ & $\mathrm{mc}$ & $\mathrm{mr}$ & $\mathrm{mc}$ & $\mathrm{mr}$ & $\mathrm{mc}$ & $\mathrm{mr}$ & sym1 & sym1 & sym1 & sym2 & sym2 & sym2 \\
\hline $\mathrm{SiO}_{2}$ & 58.15 & 55.12 & 59.48 & 55.00 & 58.81 & 56.18 & 51.72 & 51.77 & 48.25 & 52.56 & 47.68 & 50.84 \\
\hline $\mathrm{TiO}_{2}$ & 0.00 & 0.00 & 0.09 & 0.00 & 0.02 & 0.00 & 0.02 & 0.05 & 0.00 & 0.00 & 0.00 & 0.00 \\
\hline $\mathrm{Al}_{2} \mathrm{O}_{3}$ & 25.66 & 27.22 & 25.05 & 27.01 & 25.00 & 27.97 & 30.69 & 30.37 & 33.01 & 30.21 & 33.09 & 31.03 \\
\hline $\mathrm{Cr}_{2} \mathrm{O}_{3}$ & 0.05 & 0.01 & 0.04 & 0.05 & 0.07 & 0.13 & 0.02 & 0.13 & 0.00 & 0.01 & 0.00 & 0.10 \\
\hline $\mathrm{FeO}$ & 0.57 & 0.54 & 0.03 & 1.56 & 0.70 & 0.30 & 0.40 & 0.36 & 0.43 & 0.26 & 0.26 & 0.64 \\
\hline $\mathrm{MnO}$ & 0.00 & 0.00 & 0.00 & 0.00 & 0.00 & 0.00 & 0.01 & 0.00 & 0.11 & 0.11 & 0.05 & 0.00 \\
\hline $\mathrm{MgO}$ & 0.15 & 0.19 & 0.05 & 0.59 & 0.00 & 0.00 & 0.04 & 0.16 & 0.11 & 0.00 & 0.07 & 0.00 \\
\hline $\mathrm{CaO}$ & 7.57 & 9.96 & 7.10 & 10.17 & 7.71 & 10.32 & 13.52 & 13.26 & 16.49 & 13.20 & 16.53 & 14.20 \\
\hline $\mathrm{Na}_{2} \mathrm{O}$ & 6.39 & 5.36 & 7.53 & 5.43 & 6.96 & 5.61 & 3.54 & 3.63 & 1.85 & 3.85 & 2.22 & 3.20 \\
\hline $\mathrm{K}_{2} \mathrm{O}$ & 0.27 & 0.24 & 0.49 & 0.29 & 0.51 & 0.23 & 0.03 & 0.20 & 0.02 & 0.11 & 0.12 & 0.25 \\
\hline Total & 98.81 & 98.64 & 99.86 & 100.10 & 99.78 & 100.74 & 99.9999 .93 & 100.27 & 100.31 & 100.02 & 100.26 & \\
\hline An & 0.40 & 0.51 & 0.35 & 0.52 & 0.37 & 0.50 & 0.68 & 0.66 & 0.83 & 0.66 & 0.81 & 0.72 \\
\hline
\end{tabular}


Table 1 (Continued)

\begin{tabular}{|c|c|c|c|c|c|c|c|c|c|c|c|c|}
\hline \multirow[b]{2}{*}{ Sample } & \multicolumn{6}{|c|}{ Clinopyroxene } & \multicolumn{6}{|c|}{ Orthopyroxene } \\
\hline & B13 & B13 & $\mathrm{C} 75$ & $\mathrm{C} 75$ & $\mathrm{C} 13$ & $\mathrm{C} 13$ & B13 & B13 & $\mathrm{C} 75$ & C75 & $\mathrm{C} 13$ & $\mathrm{C} 13$ \\
\hline Texture $^{\mathrm{a}}$ & $\mathrm{mc}$ & $\mathrm{mr}$ & $\mathrm{mc}$ & $\mathrm{mr}$ & $\mathrm{mc}$ & $\mathrm{mr}$ & $\mathrm{mc}$ & $\mathrm{mr}$ & $\mathrm{mc}$ & $\mathrm{mr}$ & $\mathrm{mc}$ & $\mathrm{mr}$ \\
\hline $\mathrm{SiO}_{2}$ & 52.36 & 52.13 & 52.48 & 52.67 & 51.65 & 51.97 & 50.11 & 50.99 & 50.83 & 50.78 & 50.19 & 50.75 \\
\hline $\mathrm{TiO}_{2}$ & 0.04 & 0.02 & 0.11 & 0.06 & 0.06 & 0.19 & 0.00 & 0.04 & 0.05 & 0.24 & 0.06 & 0.00 \\
\hline $\mathrm{Al}_{2} \mathrm{O}_{3}$ & 1.59 & 0.67 & 0.88 & 0.89 & 1.12 & 1.07 & 0.37 & 0.45 & 0.67 & 0.51 & 0.55 & 0.75 \\
\hline $\mathrm{Cr}_{2} \mathrm{O}_{3}$ & 0.00 & 0.10 & 0.29 & 0.26 & 0.09 & 0.13 & 0.00 & 0.07 & 0.03 & 0.05 & 0.04 & 0.00 \\
\hline $\mathrm{FeO}$ & 13.42 & 13.54 & 11.77 & 11.20 & 15.25 & 13.55 & 34.94 & 32.68 & 32.11 & 32.11 & 36.01 & 33.58 \\
\hline $\mathrm{MnO}$ & 0.21 & 0.00 & 0.20 & 0.28 & 0.11 & 0.09 & 0.29 & 0.31 & 0.73 & 0.78 & 0.27 & 1.27 \\
\hline $\mathrm{MgO}$ & 11.45 & 11.55 & 11.99 & 11.70 & 10.05 & 10.85 & 12.93 & 15.11 & 14.96 & 15.04 & 12.86 & 13.90 \\
\hline $\mathrm{CaO}$ & 21.97 & 22.22 & 22.01 & 22.52 & 21.83 & 21.65 & 0.56 & 0.58 & 0.45 & 0.61 & 0.72 & 0.77 \\
\hline $\mathrm{Na}_{2} \mathrm{O}$ & 0.15 & 0.23 & 0.21 & 0.56 & 0.22 & 0.31 & 0.00 & 0.00 & 0.10 & 0.00 & 0.00 & 0.08 \\
\hline $\mathrm{K}_{2} \mathrm{O}$ & 0.07 & 0.00 & 0.00 & 0.07 & 0.00 & 0.02 & 0.00 & 0.00 & 0.09 & 0.01 & 0.00 & 0.13 \\
\hline Total & 101.26 & 100.46 & 99.94 & 100.21 & 100.38 & 99.83 & 99.20 & 100.23 & 100.22 & 100.13 & 100.70 & 101.23 \\
\hline \multirow[t]{2}{*}{$\mathrm{X}_{\mathrm{Mg}}$} & 0.6113 & 0.6272 & 0.645 & 0.6708 & 0.5474 & 0.5882 & 0.398 & 0.452 & 0.454 & 0.455 & 0.389 & 0.430 \\
\hline & \multicolumn{3}{|c|}{ Orthopyroxene } & \multicolumn{3}{|c|}{ Hornblende } & & & & & & \\
\hline Sample & 7518 & 7523 & 7517 & 7504 & 7527 & 7528 & & & & & & \\
\hline Texture $^{\mathrm{a}}$ & sym1 & sym1 & sym1 & sym2 & sym2 & sym2 & & & & & & \\
\hline $\mathrm{SiO}_{2}$ & 51.21 & 51.42 & 51.27 & 44.61 & 45.22 & 45.46 & & & & & & \\
\hline $\mathrm{TiO}_{2}$ & 0.00 & 0.07 & 0.01 & 1.24 & 1.09 & 0.79 & & & & & & \\
\hline $\mathrm{Al}_{2} \mathrm{O}_{3}$ & 1.04 & 0.85 & 1.03 & 10.72 & 11.05 & 10.51 & & & & & & \\
\hline $\mathrm{Cr}_{2} \mathrm{O}_{3}$ & 0.00 & 0.01 & 0.04 & 0.11 & 0.00 & 0.19 & & & & & & \\
\hline $\mathrm{FeO}$ & 31.26 & 31.49 & 30.59 & 19.25 & 18.20 & 18.70 & & & & & & \\
\hline $\mathrm{MnO}$ & 0.89 & 0.56 & 0.90 & 0.15 & 0.25 & 0.13 & & & & & & \\
\hline $\mathrm{MgO}$ & 15.32 & 14.83 & 15.61 & 9.98 & 10.17 & 10.41 & & & & & & \\
\hline $\mathrm{CaO}$ & 0.56 & 0.60 & 0.51 & 11.66 & 11.64 & 11.86 & & & & & & \\
\hline $\mathrm{Na}_{2} \mathrm{O}$ & 0.00 & 0.00 & 0.00 & 1.39 & 1.42 & 1.14 & & & & & & \\
\hline $\mathrm{K}_{2} \mathrm{O}$ & 0.03 & 0.00 & 0.01 & 0.87 & 0.80 & 0.62 & & & & & & \\
\hline Total & 100.31 & 99.83 & 99.97 & 99.98 & 99.84 & 99.81 & & & & & & \\
\hline $\mathrm{X}_{\mathrm{Mg}}$ & 0.466 & 0.456 & 0.476 & 0.480 & 0.499 & 0.498 & & & & & & \\
\hline
\end{tabular}

${ }^{a}$ Note: mc, matrix core; mr, matrix rim; pc, porphyroblast core; pr, porphyroblast rim; rim1, garnet rim surrounded by plagioclase + orthopyroxene symplectite; rim2, garnet rim surrounded by hornblende + plagioclase; sym1, symplectic plagioclase or orthopyroxene; sym2, plagioclase or hornblende. All analyzed samples are from the Hengshan domain. 
Table 2

Representative analyses of amphibolites

\begin{tabular}{|c|c|c|c|c|c|c|c|c|c|c|c|c|}
\hline \multirow[b]{2}{*}{ Sample } & \multicolumn{6}{|l|}{$\begin{array}{l}\text { Wutai } \\
\text { Garnet }\end{array}$} & \multicolumn{6}{|l|}{ Lüliang } \\
\hline & 0910 & 1004 & 1011 & 0909 & 1002 & 1007 & 0916 & 0918 & 1109 & 0911 & 0907 & 0935 \\
\hline Texture $^{\mathrm{a}}$ & core & core & core & rim & rim & rim & core & core & core & rim & rim & rim \\
\hline $\mathrm{SiO}_{2}$ & 37.36 & 37.06 & 37.29 & 37.90 & 37.50 & 37.17 & 37.56 & 37.65 & 37.58 & 37.43 & 37.10 & 37.51 \\
\hline $\mathrm{TiO}_{2}$ & 0.09 & 0.07 & 0.20 & 0.21 & 0.09 & 0.11 & 0.11 & 0.03 & 0.02 & 0.05 & 0.04 & 0.07 \\
\hline $\mathrm{Al}_{2} \mathrm{O}_{3}$ & 20.90 & 21.02 & 20.77 & 20.67 & 20.96 & 20.97 & 21.04 & 21.03 & 21.46 & 21.02 & 20.96 & 21.12 \\
\hline $\mathrm{FeO}$ & 26.86 & 27.62 & 26.48 & 26.77 & 26.36 & 27.62 & 30.80 & 30.58 & 26.08 & 31.37 & 32.42 & 30.79 \\
\hline $\mathrm{MnO}$ & 3.54 & 2.62 & 3.88 & 4.90 & 3.40 & 2.71 & 2.97 & 2.28 & 1.61 & 2.09 & 2.55 & 2.01 \\
\hline $\mathrm{MgO}$ & 0.84 & 1.01 & 0.81 & 1.12 & 1.01 & 1.06 & 4.53 & 4.80 & 3.29 & 4.58 & 4.13 & 4.46 \\
\hline $\mathrm{CaO}$ & 9.90 & 10.77 & 9.92 & 9.25 & 9.80 & 10.05 & 3.61 & 3.62 & 8.68 & 3.87 & 3.62 & 3.65 \\
\hline Total & 99.49 & 100.17 & 99.35 & 100.81 & 99.21 & 99.69 & 100.62 & 99.99 & 98.72 & 100.40 & 100.82 & 99.61 \\
\hline Alm & 0.599 & 0.586 & 0.592 & 0.581 & 0.579 & 0.593 & 0.661 & 0.662 & 0.583 & 0.671 & 0.688 & 0.676 \\
\hline Sps & 0.081 & 0.060 & 0.089 & 0.111 & 0.078 & 0.064 & 0.065 & 0.050 & 0.036 & 0.046 & 0.055 & 0.045 \\
\hline Prp & 0.034 & 0.041 & 0.032 & 0.045 & 0.041 & 0.044 & 0.174 & 0.187 & 0.131 & 0.176 & 0.158 & 0.176 \\
\hline \multirow[t]{3}{*}{ Grs } & 0.286 & 0.313 & 0.287 & 0.263 & 0.302 & 0.299 & 0.100 & 0.101 & 0.249 & 0.107 & 0.099 & 0.103 \\
\hline & \multicolumn{6}{|l|}{ Wutai } & \multicolumn{6}{|l|}{ Lüliang } \\
\hline & \multicolumn{12}{|c|}{ Plagioclase } \\
\hline Sample & 0910 & 1004 & 1011 & 0909 & 1002 & 1007 & 0916 & 0918 & 1109 & 0911 & 0907 & 0935 \\
\hline Texture $^{\mathrm{a}}$ & $\mathrm{mc}$ & $\mathrm{mc}$ & $\mathrm{mc}$ & sym & sym & sym & $\mathrm{mc}$ & $\mathrm{mc}$ & $\mathrm{mc}$ & sym & sym & sym \\
\hline $\mathrm{SiO}_{2}$ & 64.42 & 65.44 & 63.84 & 62.97 & 62.00 & 63.36 & 61.46 & 61.07 & 56.69 & 54.99 & 59.41 & 59.72 \\
\hline $\mathrm{TiO}_{2}$ & 0.05 & 0.08 & 0.07 & 0.04 & 0.03 & 0.01 & 0.01 & 0.06 & 0.05 & 0.10 & 0.01 & 0.04 \\
\hline $\mathrm{Al}_{2} \mathrm{O}_{3}$ & 21.99 & 21.76 & 22.36 & 22.41 & 23.52 & 21.93 & 23.86 & 24.64 & 26.64 & 28.15 & 24.43 & 25.38 \\
\hline $\mathrm{FeO}$ & 0.13 & 0.03 & 0.10 & 0.02 & 0.09 & 0.33 & 0.18 & 0.23 & 0.00 & 0.13 & 0.21 & 0.22 \\
\hline $\mathrm{MnO}$ & 0.10 & 0.07 & 0.03 & 0.13 & 0.01 & 0.06 & 0.16 & 0.09 & 0.06 & 0.03 & 0.07 & 0.08 \\
\hline $\mathrm{MgO}$ & 0.06 & 0.02 & 0.06 & 0.04 & 0.09 & 0.09 & 0.02 & 0.00 & 0.06 & 0.02 & 0.03 & 0.10 \\
\hline $\mathrm{CaO}$ & 2.88 & 2.80 & 3.23 & 7.33 & 7.59 & 8.13 & 6.12 & 7.96 & 9.67 & 10.53 & 8.33 & 8.11 \\
\hline $\mathrm{Na}_{2} \mathrm{O}$ & 10.41 & 10.18 & 10.10 & 6.06 & 6.54 & 5.30 & 8.15 & 6.84 & 6.10 & 6.14 & 6.65 & 6.99 \\
\hline $\mathrm{K}_{2} \mathrm{O}$ & 0.07 & 0.11 & 0.10 & 0.28 & 0.05 & 0.09 & 0.04 & 0.02 & 0.08 & 0.01 & 0.10 & 0.07 \\
\hline Total & 100.11 & 100.49 & 99.89 & 99.28 & 99.92 & 99.30 & 100.00 & 100.91 & 99.35 & 100.10 & 99.24 & 100.71 \\
\hline An & 13.2 & 13.2 & 14.9 & 39.4 & 39.0 & 45.6 & 29.2 & 39.1 & 46.4 & 48.6 & 40.7 & 38.9 \\
\hline
\end{tabular}


Table 2 (Continued)

\begin{tabular}{|c|c|c|c|c|c|c|c|c|c|c|c|c|}
\hline \multirow[b]{3}{*}{ Sample } & \multicolumn{6}{|l|}{ Wutai } & \multicolumn{6}{|l|}{ Lüliang } \\
\hline & \multicolumn{12}{|c|}{ Amphibole } \\
\hline & 0910 & 1004 & 1011 & 0909 & 1002 & 1007 & 0916 & 0918 & 1109 & 0911 & 0907 & 0935 \\
\hline Texture $^{\mathrm{a}}$ & $\mathrm{mc}$ & $\mathrm{mc}$ & $\mathrm{mc}$ & sym & sym & sym & $\mathrm{mc}$ & $\mathrm{mc}$ & $\mathrm{mc}$ & sym & sym & sym \\
\hline $\mathrm{SiO}_{2}$ & 39.28 & 39.39 & 37.88 & 39.97 & 39.46 & 39.95 & 45.13 & 43.45 & 45.38 & 53.72 & 54.06 & 53.56 \\
\hline $\mathrm{TiO}_{2}$ & 0.62 & 0.82 & 0.31 & 0.75 & 0.75 & 0.67 & 0.77 & 0.67 & 1.29 & 0.04 & 0.10 & 0.14 \\
\hline $\mathrm{Al}_{2} \mathrm{O}_{3}$ & 14.49 & 13.08 & 13.74 & 14.46 & 13.52 & 13.75 & 9.89 & 12.04 & 10.40 & 0.58 & 0.48 & 0.61 \\
\hline $\mathrm{FeO}$ & 23.08 & 27.30 & 27.81 & 24.21 & 26.44 & 26.48 & 21.55 & 20.61 & 17.40 & 27.67 & 26.85 & 27.51 \\
\hline $\mathrm{MnO}$ & 0.42 & 0.38 & 0.31 & 0.17 & 0.37 & 0.25 & 0.32 & 0.35 & 0.25 & 0.76 & 0.80 & 0.87 \\
\hline $\mathrm{MgO}$ & 4.96 & 4.76 & 3.15 & 5.21 & 4.44 & 4.49 & 9.73 & 9.70 & 10.59 & 15.43 & 16.08 & 15.23 \\
\hline $\mathrm{CaO}$ & 11.20 & 10.22 & 11.96 & 10.97 & 10.85 & 10.92 & 9.41 & 10.05 & 11.72 & 0.49 & 0.42 & 0.38 \\
\hline $\mathrm{Na}_{2} \mathrm{O}$ & 2.00 & 1.98 & 1.95 & 1.30 & 1.05 & 1.06 & 1.50 & 1.65 & 1.59 & 0.37 & 0.37 & 0.29 \\
\hline $\mathrm{K}_{2} \mathrm{O}$ & 1.17 & 1.27 & 1.26 & 1.14 & 1.16 & 1.17 & 0.23 & 0.35 & 0.27 & 0.06 & 0.03 & 0.09 \\
\hline Total & 97.22 & 99.20 & 98.26 & 98.18 & 98.04 & 98.75 & 98.53 & 98.87 & 98.89 & 99.13 & 99.19 & 98.68 \\
\hline \multirow[t]{3}{*}{$X_{M g}$} & 0.308 & 0.303 & 0.224 & 0.339 & 0.299 & 0.297 & 0.527 & 0.575 & 0.579 & 0.500 & 0.516 & 0.502 \\
\hline & \multicolumn{12}{|l|}{ Wutai } \\
\hline & \multicolumn{3}{|l|}{ Biotite } & \multicolumn{6}{|l|}{ Ilmenite } & & & \\
\hline Sample & 0910 & 1004 & 1011 & 0910 & 1004 & 1911 & 0909 & 1002 & 1007 & & & \\
\hline Texture $^{\mathrm{a}}$ & $\mathrm{mc}$ & $\mathrm{mc}$ & $\mathrm{mc}$ & $\mathrm{mc}$ & $\mathrm{mc}$ & $\mathrm{mc}$ & sym & sym & sym & & & \\
\hline $\mathrm{SiO}_{2}$ & 33.51 & 34.02 & 35.29 & 0.22 & 0.01 & 0.02 & 0.24 & 0.06 & 0.05 & & & \\
\hline $\mathrm{TiO}_{2}$ & 2.63 & 1.12 & 1.80 & 50.35 & 50.42 & 51.73 & 50.12 & 47.47 & 48.51 & & & \\
\hline $\mathrm{Al}_{2} \mathrm{O}_{3}$ & 16.84 & 14.08 & 15.61 & 0.05 & 0.08 & 0.07 & 0.03 & 0.07 & 0.04 & & & \\
\hline $\mathrm{FeO}$ & 27.60 & 27.09 & 25.88 & 47.55 & 48.25 & 46.24 & 47.14 & 52.52 & 51.95 & & & \\
\hline $\mathrm{MnO}$ & 0.19 & 0.01 & 0.17 & 1.13 & 1.53 & 1.91 & 1.23 & 0.15 & 0.23 & & & \\
\hline $\mathrm{MgO}$ & 6.11 & 7.94 & 6.36 & 0.04 & 0.09 & 0.05 & 0.05 & 0.00 & 0.05 & & & \\
\hline $\mathrm{CaO}$ & 0.07 & 0.01 & 0.15 & 0.16 & 0.02 & 0.09 & 0.10 & 0.07 & 0.03 & & & \\
\hline $\mathrm{Na}_{2} \mathrm{O}$ & 0.22 & 0.49 & 0.80 & 0.29 & 0.34 & 0.21 & 0.27 & 0.05 & 0.08 & & & \\
\hline $\mathrm{K}_{2} \mathrm{O}$ & 9.17 & 9.12 & 9.47 & 0.02 & 0.02 & 0.03 & 0.06 & 0.01 & 0.01 & & & \\
\hline Total & 96.35 & 93.89 & 95.54 & 99.82 & 100.76 & 100.35 & 100.24 & 100.40 & 100.96 & & & \\
\hline
\end{tabular}

a Note: mc, matrix core; sym, symplectite; $\mathrm{X}_{\mathrm{Mg}}=\mathrm{Mg} /\left(\mathrm{Mg}+\mathrm{Fe}^{2+}\right)$. Samples 0910, 1004, 1011, 0909, 1002,1007 are from the Wutai domain (Zhao et al., 1999b); samples 0916, 0918, 1109, 0911, 0907, 0935 are from the Lüliang domain. 
Table 3

Representative analyses of high-pressure granulites

\begin{tabular}{|c|c|c|c|c|c|c|c|c|c|c|c|c|}
\hline \multirow[b]{2}{*}{ Sample } & \multicolumn{12}{|l|}{ Garnet } \\
\hline & A12 & A12 & B12 & B12 & A61 & A61 & 7501 & 13310 & 13301 & 1220 & 1331 & 6014 \\
\hline Texture $^{\mathrm{a}}$ & $\mathrm{pc}$ & $\mathrm{pr}$ & $\mathrm{pc}$ & $\mathrm{pr}$ & $\mathrm{pc}$ & $\mathrm{pr}$ & rim1 & rim1 & rim1 & $\operatorname{rim} 2$ & $\operatorname{rim} 2$ & $\operatorname{rim} 2$ \\
\hline $\mathrm{SiO}_{2}$ & 38.13 & 36.77 & 38.19 & 38.01 & 38.36 & 38.27 & 38.24 & 38.29 & 37.81 & 38.30 & 38.39 & 38.67 \\
\hline $\mathrm{TiO}_{2}$ & 0.13 & 0.19 & 0.00 & 0.11 & 0.17 & 0.24 & 0.00 & 0.00 & 0.00 & 0.00 & 0.00 & 0.06 \\
\hline $\mathrm{Al}_{2} \mathrm{O}_{3}$ & 20.80 & 20.60 & 21.24 & 20.93 & 21.06 & 21.23 & 20.88 & 21.43 & 20.34 & 21.10 & 20.79 & 21.24 \\
\hline $\mathrm{Cr}_{2} \mathrm{O}_{3}$ & 0.05 & 0.00 & 0.22 & 0.00 & 0.10 & 0.04 & 0.03 & 0.08 & 0.00 & 0.03 & 0.07 & 0.03 \\
\hline $\mathrm{FeO}$ & 25.08 & 25.07 & 25.03 & 28.77 & 26.64 & 28.05 & 28.19 & 27.86 & 28.63 & 28.50 & 29.47 & 29.17 \\
\hline $\mathrm{MnO}$ & 0.75 & 2.64 & 0.55 & 0.69 & 0.48 & 1.51 & 2.03 & 0.52 & 3.38 & 2.57 & 2.98 & 1.58 \\
\hline $\mathrm{MgO}$ & 1.91 & 2.31 & 2.09 & 3.41 & 2.65 & 3.11 & 3.17 & 4.04 & 2.75 & 2.03 & 2.41 & 4.09 \\
\hline $\mathrm{CaO}$ & 13.14 & 10.40 & 13.97 & 8.74 & 11.74 & 8.73 & 7.80 & 9.19 & 6.98 & 7.47 & 7.49 & 6.87 \\
\hline Totals & 99.99 & 97.98 & 101.29 & 100.66 & 101.20 & 101.18 & 100.34 & 101.41 & 99.89 & 100.00 & 101.72 & 101.71 \\
\hline Alm & 0.540 & 0.543 & 0.512 & 0.602 & 0.559 & 0.603 & 0.613 & 0.574 & 0.616 & 0.643 & 0.630 & 0.618 \\
\hline Sps & 0.017 & 0.061 & 0.012 & 0.016 & 0.011 & 0.033 & 0.045 & 0.011 & 0.076 & 0.059 & 0.066 & 0.035 \\
\hline Prp & 0.075 & 0.093 & 0.082 & 0.135 & 0.103 & 0.121 & 0.123 & 0.157 & 0.109 & 0.082 & 0.094 & 0.158 \\
\hline Grs & 0.364 & 0.289 & 0.375 & 0.231 & 0.317 & 0.239 & 0.217 & 0.240 & 0.189 & 0.216 & 0.210 & 0.184 \\
\hline \multirow[t]{2}{*}{ Adr } & 0.014 & 0.039 & 0.056 & 0.051 & 0.033 & 0.012 & 0.006 & 0.051 & 0.031 & 0.000 & 0.020 & 0.019 \\
\hline & \multicolumn{12}{|c|}{ Plagioclase } \\
\hline Sample & A12 & A12 & B12 & B12 & A61 & A61 & 7501 & 13310 & 13101 & 1220 & 1331 & 6014 \\
\hline Texture $^{\mathrm{a}}$ & $\mathrm{mc}$ & $\mathrm{mr}$ & $\mathrm{mc}$ & $\mathrm{mr}$ & $\mathrm{mc}$ & $\mathrm{mr}$ & sym1 & corona & corona & sym2 & sym2 & sym2 \\
\hline $\mathrm{SiO}_{2}$ & 64.48 & 57.93 & 64.65 & 61.22 & 66.18 & 63.57 & 47.41 & 56.07 & 56.18 & 53.55 & 55.87 & 53.03 \\
\hline $\mathrm{TiO}_{2}$ & 0.11 & 0.00 & 0.00 & 0.09 & 0.00 & 0.00 & 0.00 & 0.00 & 0.00 & 0.03 & 0.00 & 0.00 \\
\hline $\mathrm{Al}_{2} \mathrm{O}_{3}$ & 21.73 & 25.54 & 21.67 & 24.29 & 21.15 & 22.62 & 33.37 & 26.82 & 27.97 & 30.17 & 27.52 & 28.78 \\
\hline $\mathrm{Cr}_{2} \mathrm{O}_{3}$ & 0.00 & 0.02 & 0.10 & 0.06 & 0.00 & 0.00 & 0.13 & 0.08 & 0.13 & 0.06 & 0.00 & 0.09 \\
\hline $\mathrm{FeO}$ & 0.14 & 0.31 & 0.13 & 0.18 & 0.17 & 0.01 & 0.26 & 0.46 & 0.30 & 0.33 & 0.21 & 0.44 \\
\hline $\mathrm{MnO}$ & 0.00 & 0.11 & 0.05 & 0.00 & 0.04 & 0.00 & 0.00 & 0.00 & 0.00 & 0.06 & 0.00 & 0.00 \\
\hline $\mathrm{MgO}$ & 0.00 & 0.00 & 0.06 & 0.00 & 0.07 & 0.12 & 0.00 & 0.00 & 0.00 & 0.13 & 0.02 & 0.12 \\
\hline $\mathrm{CaO}$ & 3.24 & 7.94 & 3.24 & 5.96 & 2.38 & 4.28 & 17.36 & 9.58 & 10.32 & 12.56 & 10.10 & 12.02 \\
\hline $\mathrm{Na}_{2} \mathrm{O}$ & 9.01 & 6.74 & 9.15 & 7.70 & 9.49 & 8.83 & 1.63 & 5.35 & 5.61 & 4.29 & 5.30 & 3.98 \\
\hline $\mathrm{K}_{2} \mathrm{O}$ & 0.27 & 0.41 & 0.02 & 0.42 & 0.69 & 0.35 & 0.08 & 0.26 & 0.23 & 0.06 & 0.14 & 0.23 \\
\hline Totals & 98.98 & 99.00 & 99.07 & 99.92 & 100.17 & 99.78 & 100.24 & 98.62 & 100.74 & 101.24 & 99.16 & 98.69 \\
\hline $\mathrm{An}$ & 0.16 & 0.39 & 0.16 & 0.29 & 0.12 & 0.21 & 0.85 & 0.49 & 0.50 & 0.62 & 0.52 & 0.63 \\
\hline
\end{tabular}


Table 3 (Continued)

\begin{tabular}{|c|c|c|c|c|c|c|c|c|c|c|c|c|}
\hline \multirow[b]{2}{*}{ Sample } & \multicolumn{6}{|c|}{ Clinopyroxene } & \multicolumn{6}{|c|}{ Hornblende } \\
\hline & A12 & A12 & $\mathrm{B} 12$ & $\mathrm{~B} 12$ & A61 & A61 & A12 & A12 & $\mathrm{B} 12$ & B12 & A61 & A61 \\
\hline Texture $^{\mathrm{a}}$ & $\mathrm{mc}$ & $\mathrm{mr}$ & $\mathrm{mc}$ & $\mathrm{mr}$ & $\mathrm{mc}$ & $\mathrm{mr}$ & $\mathrm{mc}$ & $\mathrm{mr}$ & $\mathrm{mc}$ & $\mathrm{mr}$ & $\mathrm{mc}$ & $\mathrm{mr}$ \\
\hline $\mathrm{SiO}_{2}$ & 51.77 & 51.43 & 52.41 & 51.68 & 53.37 & 52.44 & 41.97 & 40.40 & 44.45 & 43.24 & 42.50 & 41.04 \\
\hline $\mathrm{TiO}_{2}$ & 0.01 & 0.16 & 0.15 & 0.15 & 0.00 & 0.12 & 1.91 & 1.13 & 1.87 & 1.13 & 1.77 & 1.23 \\
\hline $\mathrm{Al}_{2} \mathrm{O}_{3}$ & 1.41 & 1.19 & 0.81 & 0.67 & 1.11 & 0.81 & 11.87 & 13.68 & 8.82 & 14.20 & 11.74 & 12.67 \\
\hline $\mathrm{Cr}_{2} \mathrm{O}_{3}$ & 0.00 & 0.01 & 0.17 & 0.23 & 0.16 & 0.00 & 0.05 & 0.00 & 0.00 & 0.18 & 0.23 & 0.27 \\
\hline $\mathrm{FeO}$ & 13.98 & 13.90 & 13.50 & 11.91 & 11.24 & 11.55 & 19.27 & 20.58 & 18.65 & 17.06 & 19.28 & 19.29 \\
\hline $\mathrm{MnO}$ & 0.17 & 0.46 & 0.17 & 0.13 & 0.24 & 0.35 & 0.07 & 0.10 & 0.14 & 0.03 & 0.07 & 0.15 \\
\hline $\mathrm{MgO}$ & 11.08 & 10.59 & 11.38 & 11.90 & 12.55 & 11.82 & 9.11 & 7.72 & 9.43 & 7.13 & 9.65 & 8.38 \\
\hline $\mathrm{CaO}$ & 21.19 & 21.58 & 21.64 & 21.11 & 22.64 & 22.65 & 11.41 & 11.22 & 12.74 & 10.43 & 11.54 & 11.52 \\
\hline $\mathrm{Na}_{2} \mathrm{O}$ & 0.38 & 0.00 & 0.04 & 0.11 & 0.40 & 0.25 & 1.68 & 1.35 & 1.37 & 2.02 & 1.87 & 1.21 \\
\hline $\mathrm{K}_{2} \mathrm{O}$ & 0.04 & 0.00 & 0.03 & 0.18 & 0.00 & 0.03 & 1.36 & 1.57 & 1.01 & 2.14 & 1.20 & 1.55 \\
\hline Totals & 100.03 & 99.32 & 100.30 & 98.07 & 101.71 & 100.02 & 98.70 & 97.75 & 98.48 & 97.56 & 99.85 & 97.31 \\
\hline \multirow[t]{2}{*}{$\mathrm{X}_{\mathrm{Mg}}$} & 0.603 & 0.575 & 0.600 & 0.640 & 0.684 & 0.654 & 0.457 & 0.400 & 0.474 & 0.427 & 0.471 & 0.436 \\
\hline & \multicolumn{3}{|c|}{ Orthopyroxene } & & \multicolumn{3}{|c|}{ Hornblende } & & \multicolumn{4}{|c|}{ Clinopyroxene } \\
\hline Sample & 7501 & 13310 & 13101 & & 1220 & 1331 & 6014 & & 13310 & 13101 & 7511 & 7531 \\
\hline Texture $^{\mathrm{a}}$ & sym & corona & corona & & sym2 & sym2 & sym2 & & corona & corona & corona & corona \\
\hline $\mathrm{SiO}_{2}$ & 51.04 & 50.22 & 50.74 & & 42.32 & 40.89 & 47.42 & & 51.81 & 52.02 & 52.09 & 52.16 \\
\hline $\mathrm{TiO}_{2}$ & 0.05 & 0.11 & 0.11 & & 1.92 & 1.31 & 0.45 & & 0.12 & 0.09 & 0.22 & 0.04 \\
\hline $\mathrm{Al}_{2} \mathrm{O}_{3}$ & 0.79 & 0.75 & 0.58 & & 11.06 & 12.88 & 9.03 & & 1.06 & 0.84 & 1.23 & 0.62 \\
\hline $\mathrm{Cr}_{2} \mathrm{O}_{3}$ & 0.15 & 0.03 & 0.16 & & 0.03 & 0.02 & 0.15 & & 0.10 & 0.00 & 0.05 & 0.00 \\
\hline $\mathrm{FeO}$ & 31.89 & 34.01 & 35.03 & & 18.52 & 18.76 & 17.21 & & 13.94 & 14.53 & 12.30 & 12.15 \\
\hline $\mathrm{MnO}$ & 0.63 & 1.23 & 0.19 & & 0.09 & 0.20 & 0.09 & & 0.43 & 0.02 & 0.17 & 0.18 \\
\hline $\mathrm{MgO}$ & 14.88 & 13.62 & 13.68 & & 8.98 & 8.34 & 11.71 & & 11.70 & 10.88 & 11.80 & 11.71 \\
\hline $\mathrm{CaO}$ & 0.63 & 0.56 & 0.68 & & 11.29 & 11.24 & 11.40 & & 21.39 & 21.70 & 22.10 & 22.23 \\
\hline $\mathrm{Na}_{2} \mathrm{O}$ & 0.00 & 0.00 & 0.00 & & 1.60 & 1.45 & 0.91 & & 0.24 & 0.00 & 0.17 & 0.01 \\
\hline $\mathrm{K}_{2} \mathrm{O}$ & 0.02 & 0.00 & 0.00 & & 1.17 & 1.67 & 0.67 & & 0.03 & 0.00 & 0.15 & 0.00 \\
\hline Totals & 100.08 & 100.53 & 101.17 & & 96.98 & 96.76 & 99.04 & & 100.82 & 100.08 & 100.28 & 99.10 \\
\hline $\mathrm{X}_{\mathrm{Mg}}$ & 0.454 & 0.417 & 0.410 & & 0.463 & 0.441 & 0.548 & & 0.6317 & 0.5717 & 0.6422 & 0.6322 \\
\hline
\end{tabular}

a Note: mc, matrix core; mr, matrix rim; pc, porphyroblast core; pr, porphyroblast rim; rim1, garnet rim surrounded by plagioclase + orthopyroxene symplectite;

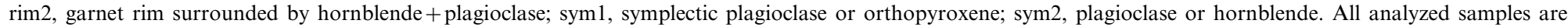
from the Hengshan high-pressure granulites. 
Although there are sharp contacts between the mafic granulites and the TTG gneisses, no obvious intrusive relationships have been observed. The long axes of the granulite boudins are always parallel to the regional strike of the foliation of the TTG gneisses. What remains unknown is the protoliths of the mafic granulites; they may have been derived from either metamorphosed basalts or from metamorphosed basic intrusives, including gabbros and dolerite dykes. Two age groups have been recognized from cores and rims of zircons by SHRIMP $\mathrm{U}-\mathrm{Pb}$ dating of a TTG gneiss sample; the older cores at $\sim 2550 \mathrm{Ma}$, interpreted to represent the protolith age, and the younger rims at $\sim 1800 \mathrm{Ma}$, corresponding to the age of the subsequent, overprinting metamorphic event (Wilde et al., 1998; Kröner, personal communication, 1999).

Petrographic observations in the garnetiferous mafic granulites indicate three stages of metamorphic evolution. The $\mathrm{M}_{1}$ stage represents the growth of relatively coarse-grained garnet + orthopyroxene + clinopyroxene + plagioclase + quartz. The minerals in this stage display granoblastic polygonal texture. Garnet occurs mainly as inclusionfree, porphyroblast grains. Chemically, garnets are grossular- and pyrope-rich and spessartine-poor almandine $\left(\mathrm{Alm}_{53-65} \mathrm{Grs}_{16-32} \operatorname{Prp}_{12-15} \mathrm{Sps}_{1-6}\right.$; Table 1). The zoning profile of garnet is characterized by a decrease in almandine and an increase in grossular from rim to core, whereas spessartine and pyrope contents are nearly uniform (Table 1 and Fig. 4(a)). Orthopyroxene, clinopyroxene, plagioclase and quartz occur as matrix minerals. Plagioclase shows a distinct compositional zoning, with an andesine core and a labradorite rim (Table 1 and Fig. 4(b)), whereas matrix-type clinopyroxene and orthopyroxene lack any pronounced compositional zoning.
The $\mathrm{M}_{2}$ stage is represented by worm-like hypersthene + plagioclase \pm magnetite symplectites (Fig. 5(a)) or clinopyroxene + orthopyroxene + plagioclase coronas that commonly separate garnet from quartz grains (Fig. 5(b)). Locally, orthopyroxene + plagioclase \pm magnetite symplectites completely replace garnet grains and occur as pseudomorphs (Fig. 5(c)). These textures suggest a combination of the following decompressional reactions (Harley, 1989):

garnet + quartz $\rightarrow$ orthopyroxene + plagioclase

garnet + quartz $+\mathrm{O}_{2}$

$\rightarrow$ orthopyroxene + plagioclase + magnetite

garnet + quartz $\rightarrow$ clinopyroxene + plagioclase

The $\mathrm{M}_{3}$ stage is represented by hornblende + plagioclase symplectites around garnet grains (Fig. 5(d)). In most cases, hornblende + plagioclase symplectites in the granulites occur around those garnet grains which lack pyroxene + plagioclase symplectites; or coronas. In some places, however, the two kinds of symplectites or coronas coexist near the same garnet grain. In the latter case, the hornblende + plagioclase symplectites or coronas are invariably located adjacent to the garnet grain and separate the embayed garnet grains from the pyroxene + plagioclase symplectites or coronas (Fig. 5(c)). Locally, the symplectic orthopyroxene and/or clinopyroxene grains are replaced by symplectic hornblende (Fig. 5(f)). These textures suggest that the hornblende + plagioclase symplectite formed later than the pyroxene + plagioclase symplectites or coronas. The formation of hornblende + plagioclase has been related to the following two generalized hydration

Fig. 4. Representative mineral compositional zoning profiles [a-b, Hengshan (HS) domain; $\mathrm{c}-\mathrm{h}$, HPG domain]. (a) Garnet: rim to rim in medium-pressure granulites (next to plagioclase + quartz at both rims). (b) Matrix-type plagioclase grains between quartz (right) and clinopyroxene (left) in medium-pressure granulites. (c) Garnet: (rim to rim) in high-pressure granulites (next to clinopyroxene + orthopyroxene + plagioclase corona at both rims). (d) A matrix-type plagioclase grain between clinopyroxene grains in high-pressure granulite. (e) A matrix-type clinopyroxene grain between plagioclase (right) and quartz (left) in high-pressure granulites. (f) A matrix-type hornblende grain between plagioclase (right) and clinopyroxene (left) in high-pressure granulites. (g) A symplectic plagioclase grain associated with orthpyroxene as a pseudomorph after garnet porphyroblasts. (h) A symplectic orthopyroxene grain associated with plagioclase as a pseudomorph after garnet porphyroblasts in high-pressure granulites. 

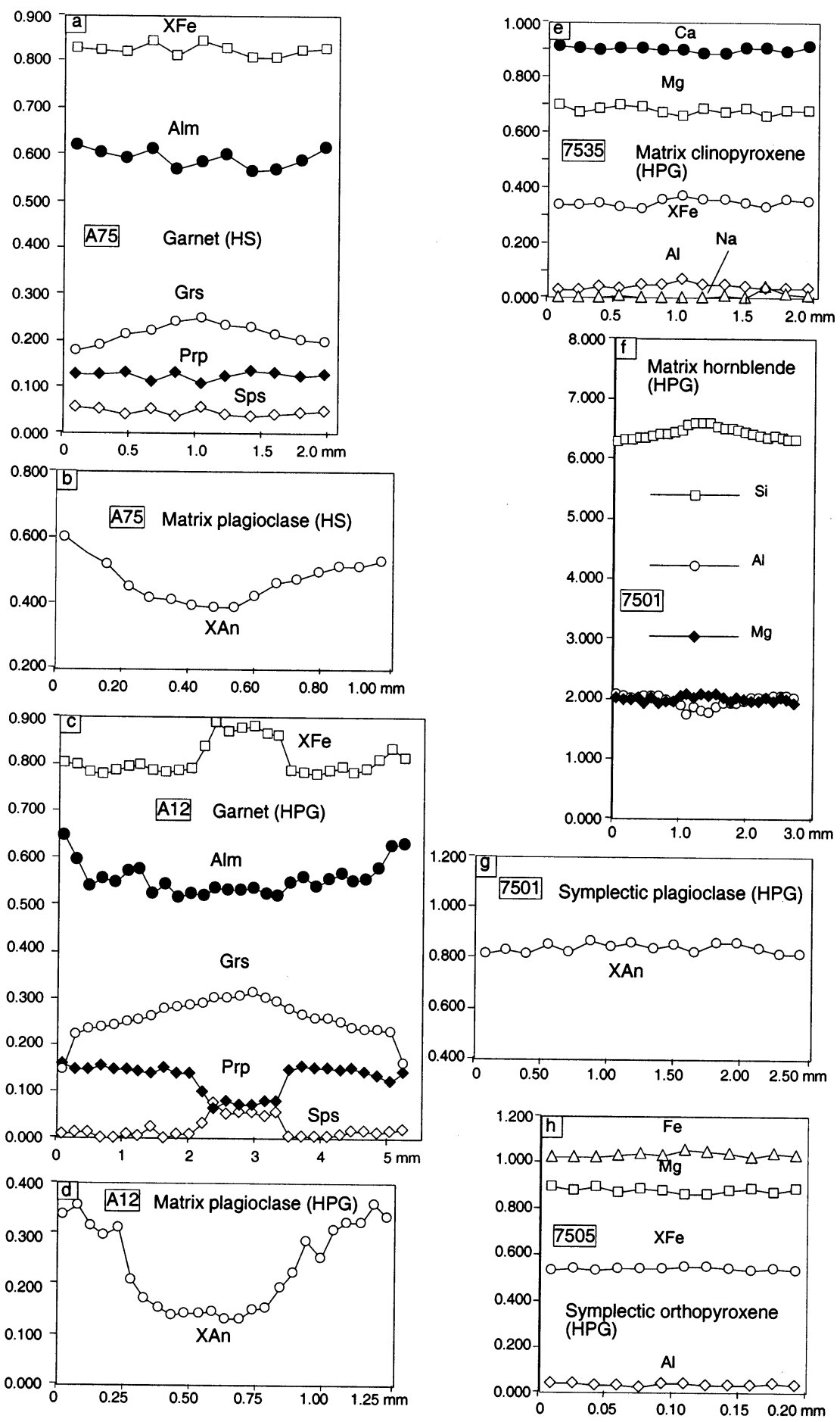

Fig. 4. 
reactions by Harley (1989) and Mengel and Rivers (1991):

garnet + clinopyroxene + quartz $+\mathrm{H}_{2} \mathrm{O} \pm \mathrm{O}_{2}$

$\rightarrow$ plagioclase + hornblende \pm magnetite

garnet $_{1}+$ plagioclase $_{1}+$ quartz $+\mathrm{H}_{2} \mathrm{O}$

$\rightarrow$ garnet $_{2}+$ plagioclase $_{2}+$ hornblende

Both reactions require the presence of some water, suggesting that their occurrence depends on fluid compositions as well as $\mathrm{P}-\mathrm{T}$ conditions.
The program THERMOCALC (Powell and Holland, 1994) was used to estimate the metamorphic $\mathrm{P}-\mathrm{T}$ conditions of the $\mathrm{M}_{1}, \mathrm{M}_{2}$ and $\mathrm{M}_{3}$ stages because this program contains thermodynamic data for Ca-tschermak and Mg-tschermak's pyroxenes which are significant components in pyroxenes of the Hengshan granulites. Appendix A gives details about this program, including the internally consistent thermodynamic databasets and activity models used in the program.
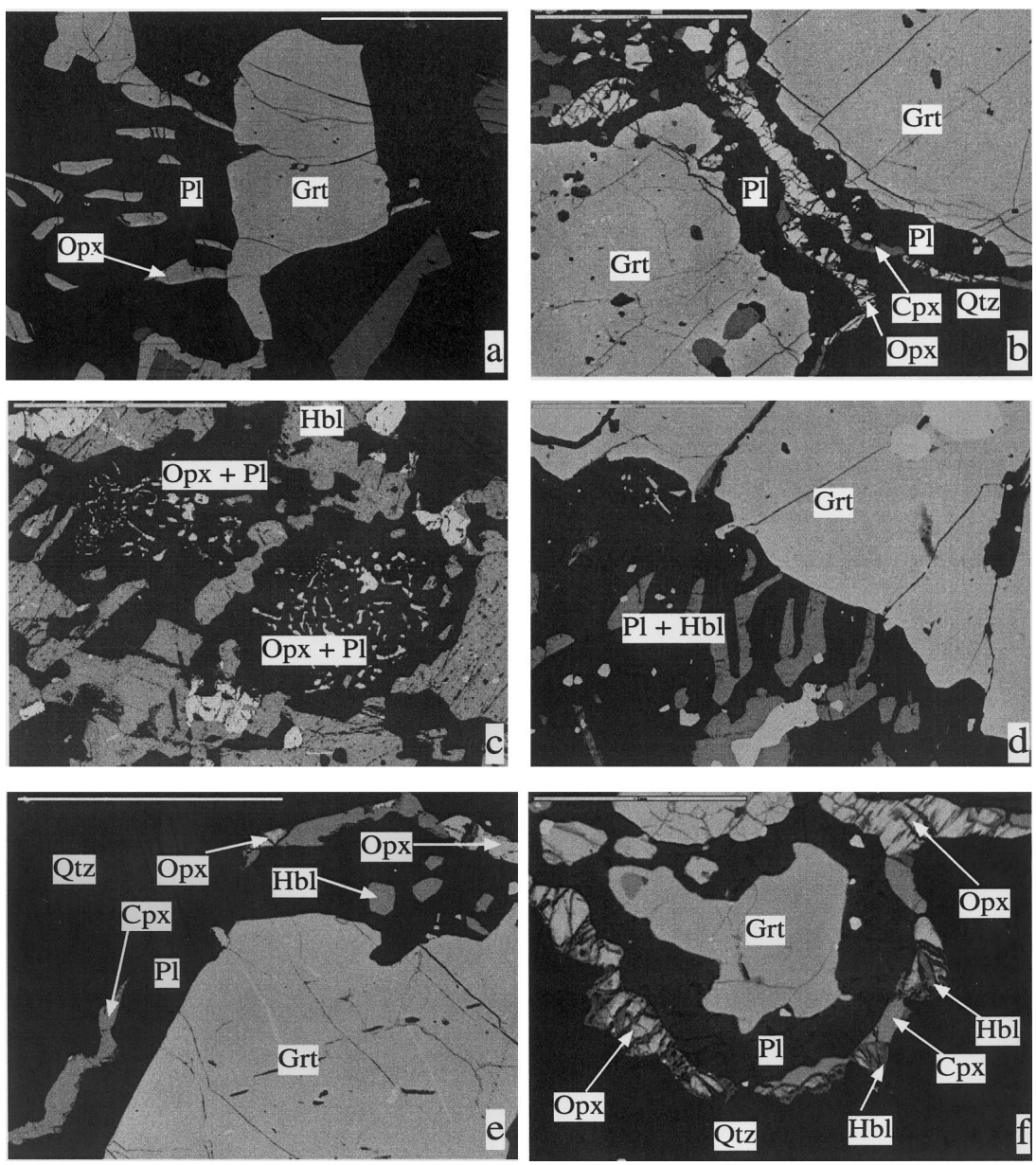

Fig. 5. Back-scattered electron images showing representative metamorphic reaction textures of medium-pressure granulites. Scale bar: $1 \mathrm{~mm}$. (a) Plagioclase + orthopyroxene symplectite around garnet. (b) Plagioclase + clinopyroxene + orthopyroxene corona surrounding garnet. (c) Plagioclase + orthopyroxene symplectite as garnet pseudomorph after garnet. (d) Hornblende + plagioclase symplectite around garnet. (e) Orthopyroxene + clinopyroxene + plagioclase corona mantles hornblende + plagioclase symplectite surrounding garnet. (f) Clinopyroxene + orthpyroxene corona replaced by hornblende. All images were collected from the medium-pressure granulites in the Hengshan domain. Mineral symbols are after Kreze (1983). 
To reduce the effect of resetting during postpeak thermal events $\left(\mathrm{M}_{2}, \mathrm{M}_{3}\right)$, the estimates of $\mathrm{P}-\mathrm{T}$ conditions during the peak stage $\left(\mathrm{M}_{1}\right)$ are based on the core compositions of garnet and matrix orthopyroxene, clinopyroxene and plagioclase from those samples which do not contain symplectites. The end-member phases that can best reflect the bulk composition and record the metamorphic conditions of the $\mathrm{M}_{1}$ assemblage include anorthite, pyrope, almandine, grossular, quartz, diopside, hedenbergite, Ca-tschermak pyroxene, ferrosilite, enstatite and Mg-tschermak's pyroxene, which make up six independent equilibria. The average $\mathrm{P}-\mathrm{T}$ conditions estimated with these independent equilibria are 9-11 kbar and $820-870^{\circ} \mathrm{C}$ for the core compositions, and $7.0-$ $8.0 \mathrm{kbar}$ and $730-850^{\circ} \mathrm{C}$ for the rim compositions, with the standard errors of $\pm 0.5-1.5 \mathrm{kbar}$ and $\pm 20-60^{\circ} \mathrm{C}$. The former is assumed to represent the peak $\mathrm{P}-\mathrm{T}$ conditions, whereas the latter represents $\mathrm{P}-\mathrm{T}$ conditions during $\mathrm{M}_{2}$ and/or $\mathrm{M}_{3}$.

The $\mathrm{P}-\mathrm{T}$ conditions of $\mathrm{M}_{2}$ are difficult to estimate because the existence of a corona reflects a disequilibrium between the minerals inside, outside and across the corona. However, local equilibrium between the minerals is assumed since symplectic and coronitic minerals do not show pronounced compositional zoning. The $\mathrm{P}-\mathrm{T}$ estimates for the $\mathbf{M}_{2}$ assemblage are based on garnet and symplectic/coronitic clinopyroxene, orthopyroxene and plagioclase compositions just inside the outermost rims $(30-50 \mu \mathrm{m})$, assuming that these compositions have been in equilibrium. For the orthopyroxene + clinopyroxene + plagioclase corona, the end-member phases and their solid solution models were the same as those used for the peak assemblage $\left(\mathrm{M}_{1}\right)$. For the orthopyroxene + plagioclase symplectite, the selected endmember phases include anorthite, pyrope, almandine, grossular, quartz, ferrosilite, enstatite and Mg-tschermak's pyroxene and THERMOCALC yielded average $\mathrm{P}-\mathrm{T}$ conditions of 6.5-7.5 kbar and $740-840^{\circ} \mathrm{C}$ for the $\mathrm{M}_{2}$ stage, with the standard errors of $\pm 0.5-1.0$ kbar and $\pm 20-$ $50^{\circ} \mathrm{C}$.

The compositions of garnet, symplectic amphibole and plagioclase rims inside the outermost rims were selected for determination of $\mathrm{P}-\mathrm{T}$ con- ditions of the $\mathrm{M}_{3}$ stage. The end-member phases chosen were anorthite, albite, pyrope, almandine, grossular, quartz, tremolite, tschermakite, Fe-actinolite, pargasite, glaucophane and $\mathrm{H}_{2} \mathrm{O}$, which constitute five independent reactions. The $\mathrm{M}_{3} \mathrm{P}-$ $\mathrm{T}$ conditions estimated with these five independent reactions by THERMOCALC fall in the range $4.0-6.0 \mathrm{kbar}$ and $680-750^{\circ} \mathrm{C}$, with the standard errors of $\pm 0.5-1.0 \mathrm{kbar}$ and $\pm 20-40^{\circ} \mathrm{C}$.

Taken together, the textural relations and the quantitative $\mathrm{P}-\mathrm{T}$ estimates for the $\mathrm{M}_{1}, \mathrm{M}_{2}$ and $\mathrm{M}_{3}$ assemblages define a nearly isothermal decompression clockwise $\mathrm{P}-\mathrm{T}$ path for the mafic granulites from the Hengshan domain (Fig. 6(a)).

\subsection{Wutai domain (WT)}

The Wutai domain, bounded to the northwest by the Hengshan domain and to the southeast by the Fuping domain, represents the best preserved granite-greenstone terrane in the North China Craton (Fig. 2; Bai, 1986; Bai et al., 1992; Tian, 1991). The domain comprises a sequence of metamorphosed ultramafic to felsic volcanic rocks, variably deformed granitoid rocks, along with lesser amounts of siliciclastic and carbonate rocks and banded iron formations. SHRIMP ages of zircons from greenstone rocks and associated granitoid rocks from the Wutai domain range from 2550 to $2520 \mathrm{Ma}$ (Wilde et al., 1997). The age of the peak metamorphism has not been well constrained, but a minimum age is provided by hornblendes from an amphibolite sample which have been dated using the ${ }^{40} \mathrm{Ar} /{ }^{39} \mathrm{Ar}$ method at $1781 \pm 20 \mathrm{Ma}$, interpreted as a cooling age (Wang et al., 1997a).

Petrographic evidence from the Wutai amphibolites shows four metamorphic stages. The $\mathbf{M}_{1}$ assemblage is composed of plagioclase + quartz + actinolite + chlorite + epidote + biotite + rutile, preserved as inclusions within garnet porphyroblasts. This is a greenschist facies assemblage which is stable between 400 and $500^{\circ} \mathrm{C}$ (cf. Yardley, 1989), whereas its pressure cannot be qualitatively estimated because of the lack of suitable mineral assemblages. Considering the presence of rutile, which has the smallest molecular volume of the $\mathrm{TiO}_{2}$ polymorphs and tends to occur in relatively 


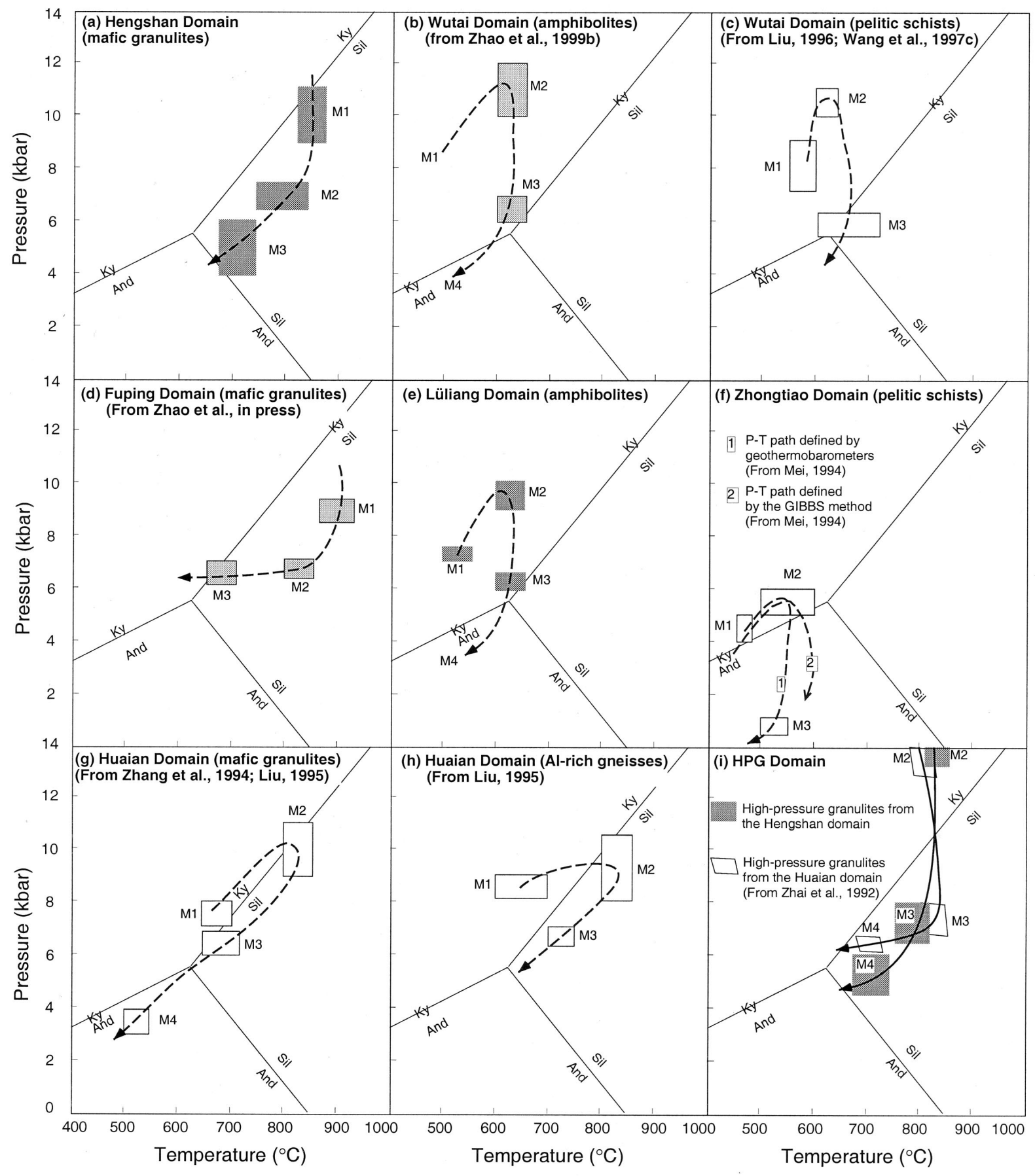

P-T estimated by THERMOCALC

P-T estimated by TWQ

P-T estimated by conventional thermobarometry combined with petrogenetic grids

Fig. 6. Metamorphic $\mathrm{P}-\mathrm{T}$ paths of the basement rocks in the Central Zone of the North China craton. Mineral symbols are after Kreze (1983). $\mathrm{Al}_{2} \mathrm{SiO}_{5}$ fields are after Holdaway and Murkhopadhyay (1993) 

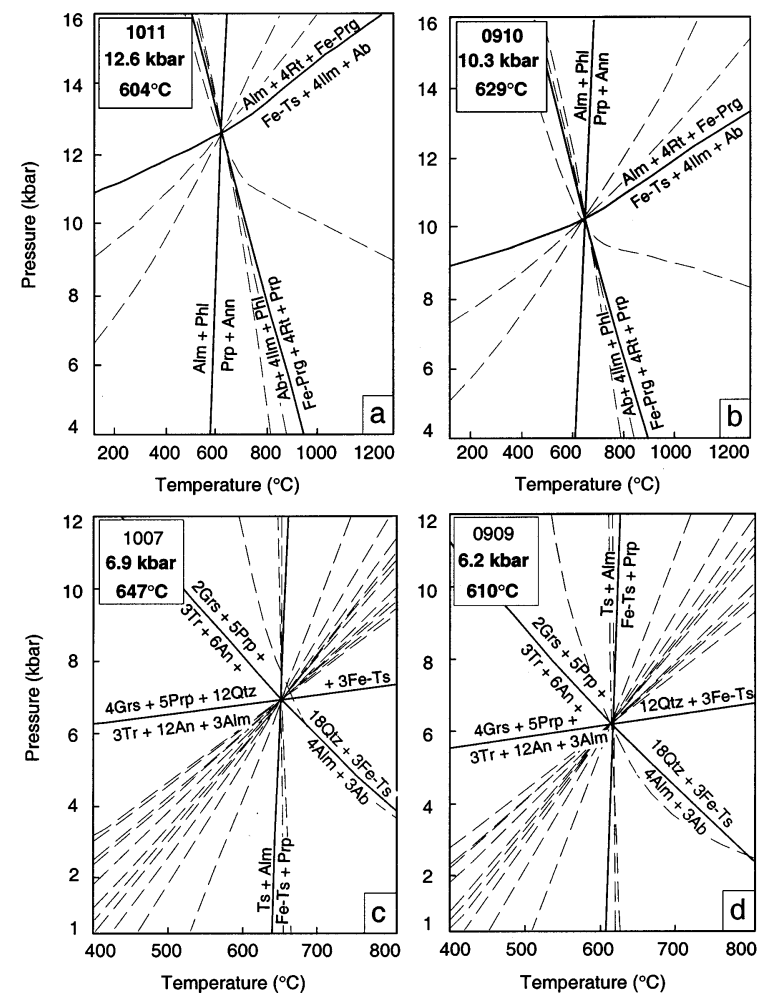

Fig. 7. Representative TWQ plots for the $\mathbf{M}_{2}(\mathrm{a}, \mathrm{b})$ and $\mathbf{M}_{3}$ (c, d) stages of amphibolites from the Wutai domain. Mineral symbols are after Kreze (1983).

high-pressure assemblages (Deer et al., 1992), the pressure of $M_{1}$ is assumed to be higher than 3-4 kbar. The $\mathbf{M}_{2}$ stage represents the growth of coarse garnet porphyroblasts and matrix minerals of amphibole + plagioclase + quartz + biotite \pm clinopyroxene \pm rutile \pm ilmenite. Garnet occurs as subhedral porphyroblasts (up to $3.0 \mathrm{~mm}$ ) containing $\mathrm{M}_{1}$ mineral inclusions, and are grossularrich, relatively pyrope- and spessartine-poor almandine, without pronounced compositional variations from core to rim (Table 2). Plagioclase and quartz occur primarily as xenoblastic grains $(0.5-1.0 \mathrm{~mm})$. Plagioclases are Na-rich and range from $\mathrm{An}_{11}$ to $\mathrm{An}_{20}$ (Table 2), without compositional zoning. Amphibole occurs as subhedral grains $(0.5-1.5 \mathrm{~mm})$, and ranges modally from 30 to $70 \%$. Biotite and ilmenite appear commonly in minor amounts, with little compositional variation from core to rim. The $\mathrm{P}-\mathrm{T}$ conditions of $\mathrm{M}_{2}$ were estimated using the TWQ program (Berman, 1991), based on the core compositions of garnet porphyroblast and matrix amphibole, plagioclase, ilmenite and biotite. Appendix A gives a detailed description about this program, including its main differences from the THERMOCALC program and the internally consistent thermodynamic databasets and activity models used in this program. The end-member phases used in calculations for the $\mathrm{M}_{2}$ stage are albite, anorthite, pyrope, almandine, beta-quartz, Fe-tremolite, Fe-tschermakite, Fe-pargasite, phlogopite, annite, ilmenite and rutile. The choice of the Fe-bearing amphibole end-members was based on the fact that the compositions of the matrix amphibole $\left(\mathrm{M}_{2}\right)$ vary from ferroan-pargasite to ferro-pargasite, and to hastingsite. Eight equilibria can be written between the selected end-member phases, of which three are linearly independent. Two representative TWQ results are presented graphically in Fig. $7(a-b)$, which show a good intrasample convergence of the eight possible equilibria (three independent). The average $\mathrm{P}-\mathrm{T}$ conditions for the $\mathrm{M}_{2}$ stage are $600-650^{\circ} \mathrm{C}$ and $10-12 \mathrm{kbar}$.

The $\mathrm{M}_{3}$ stage is represented by amphibole + plagioclase, ilmenite + plagioclase and ilmenite + plagioclase + amphibole symplectites around the embayed garnet grains. Symplectic amphibole occurs as fine subhedral grains, intergrown with symplectic plagioclase. They may have been produced by the following generalized reactions: garnet (core) + plagioclase (matrix)

+ quartz (matrix or inclusion) $+\mathrm{H}_{2} \mathrm{O}$

$\rightarrow$ garnet $($ rim $)+$ plagioclase (symplectite)

+ amphibole (symplectite)

garnet + plagioclase (matrix) + rutile (matrix)

$\rightarrow$ plagioclase (symplectite)

+ ilmenite (symplectite)

where in both cases symplectic plagioclase is more An-rich than matrix plagioclase. For example, An content is between 40 and 45 for symplectic plagioclase and between 13 and 15 for matrix plagioclase (Table 2). Compared with the matrix-type amphibole, symplectic amphibole is lower in $\mathrm{Na}_{2} \mathrm{O}$ and $\mathrm{FeO}$ (Table 2). The $\mathrm{P}-\mathrm{T}$ conditions of the $\mathrm{M}_{3}$ stage were estimated based on the rim 
compositions of garnet and symplectic amphibole and plagioclase. The end-member phases used in the calculations were albite, anorthite, pyrope, almandine, grossular, beta-quartz, tremolite, tschermakite, Fe-tschermakite and Fe-pargasite, which constitute 17 possible equilibria (three independent). Two representative TWQ results are shown in Fig. 7(c-d). The TWQ results are quite similar and yield an average temperature of 610 $650^{\circ} \mathrm{C}$ and pressure of $6.0-7.0 \mathrm{kbar}$ for the $\mathrm{M}_{3}$ stage.

The $\mathrm{M}_{4}$ assemblage is represented by chlorite and epidote replacing garnet, chlorite replacing amphibole and epidote replacing plagioclase. These replacements represent a retrogressive or retrograde metamorphic process involving the breakdown of relatively higher $\mathrm{P}-\mathrm{T}$ assemblages in association with declining $\mathrm{P}-\mathrm{T}$ conditions (Barker, 1989, pp. 60-62). Therefore, the $\mathrm{P}-\mathrm{T}$ estimates from the four metamorphic stages for the Wutai domain define a isothermal decompressional, clockwise, $\mathrm{P}-\mathrm{T}$ path (Fig. 6(b)).

Petrographic observations in the Wutai pelitic gneisses or schists indicate dime metamorphic mineral assemblages. The early prograde assemblage $\left(\mathrm{M}_{1}\right)$ is plagioclase + quartz + biotite + muscovite + staurolite, occurring as inclusions within garnet porphyroblasts. The peak assemblage is plagioclase + quartz + kyanite/sillimanite + biotite + garnet \pm muscovite, representing the growth of porphyroblasts and matrix minerals. The decompressional assemblage is represented by cordierite + plagioclase symplectites surrounding garnet porphyroblasts (Liu, 1996b; Wang et al., 1997c). The $\mathrm{P}-\mathrm{T}$ conditions of $\mathrm{M}_{1}$ were estimated at $7-8 \mathrm{kbar}$ and $550-600^{\circ} \mathrm{C}$, based on the garnet-biotite-muscovite-plagioclase thermobarometers of Hodges and Crowley (1985). The $\mathrm{P}-\mathrm{T}$ conditions of $\mathrm{M}_{2}$ were estimated at $10-11 \mathrm{kbar}$ and $600-650^{\circ} \mathrm{C}$, with the garnetbiotite thermometer of Hodges and Crowley (1985) and the garnet-plagioclase- $\mathrm{Al}_{2} \mathrm{SiO}_{5}-$ quartz barometer of Koziol and Newton (1988). The $\mathrm{P}-\mathrm{T}$ conditions of $\mathrm{M}_{3}$ were estimated at $10-11$ kbar and $600-650^{\circ} \mathrm{C}$, based on the garnet-cordierite thermometer of Aranovich and Kosyakova (1983) and the garnet-cordierite-sillimanite-quartz barometer of Aranovich and
Kosyakova (1983). These $\mathrm{P}-\mathrm{T}$ estimates also define a isothermal decompressional, clockwise, P-T path (Fig. 6(c); Liu, 1996a; Wang et al., 1997c).

\subsection{Fuping domain (FP)}

The Fuping domain lies adjacent to the southeastern part of the Wutai domain and approximately $250 \mathrm{~km}$ southwest of Beijing (Fig. 2). It consists predominantly of a variety of grey gneisses, mafic granulites, amphibolites and metapelites, banded iron formation, calcsilicate rocks and marbles in amphibolite to granulite facies (Zhao et al., in press). SHRIMP $\mathrm{U}-\mathrm{Pb}$ zircon dating of the TTG gneisses from the Fuping domain reveal two distinct age groups at 2750-2500 and $\sim 1800 \mathrm{Ma}$ (Wilde et al., 1998). The older ages were obtained in the cores of zircons and are interpreted to be the protolith age, whereas the younger ages come from rims of zircons and are interpreted to represent a metamorphic event (Wilde et al., 1998).

The mafic granulites preserve peak and peakpost metamorphic reaction textures and $\mathrm{P}-\mathrm{T}$ information. Like the mafic granulites from the Hengshan domain, the mineral assemblages in the Fuping mafic granulites reflect three metamorphic stages (Zhao et al., in press). The $\mathrm{M}_{1}$ stage is represented by the growth of garnet porphyroblasts and matrix quartz + plagioclase + orthopyroxene + clinopyroxene; $\mathrm{M}_{2}$ is documented by worm-like orthopyroxene + clinopyroxene + plagioclase \pm magnetite symplectites and coronas around embayed garnet grains; and $\mathrm{M}_{3}$ is represented by hornblende + plagioclase symplectites surrounding garnet grains. The symplectic and coronitic plagioclase, orthopyroxene and clinopyroxene were produced through reactions (1), (2) and (3), and the symplectic hornblende and plagioclase were produced by hydration reactions (4) and (5). The $\mathrm{M}_{1} \mathrm{P}-\mathrm{T}$ conditions obtained with TWQ thermobarometry are 8.5-9.5 kbar and $870-930^{\circ} \mathrm{C}$ for the $M_{1}$ assemblage, based on the core compositions of garnet, matrix orthopyroxene, clinopyroxene and plagioclase. The $\mathrm{P}-\mathrm{T}$ conditions of $\mathrm{M}_{2}$ were estimated at 6.5-7.0 kbar and $800-850^{\circ} \mathrm{C}$ based on the garnet rim compositions 
and symplectic or coronitic orthopyroxene, clinopyroxene and plagioclase compositions. The $\mathrm{M}_{3} \mathrm{P}-\mathrm{T}$ conditions were calculated at $6.0-7.0$ kbar and $650-700^{\circ} \mathrm{C}$ based on garnet rim compositions and symplectic hornblende and plagioclase compositions. These $\mathrm{P}-\mathrm{T}$ estimates define a nearisothermal decompressional, clockwise $\mathrm{P}-\mathrm{T}$ path for the Fuping domain (Fig. 6(d)). Similar P-T paths were determined from the pelitic gneisses in the Fuping domain (Liu and Liang, 1997).

\subsection{Lüliang domain (LL)}

The Lüliang domain is located in the western Shanxi Province, approximately $200 \mathrm{~km}$ southwest of the Wutai domain (Fig. 2). The domain consists of a lower sequence (the Jiehekou Group) of paragneisses, amphibolites and carbonates metamorphosed at amphibolite facies, and a tectonically overlying sequence (the Lüliang Group) composed of clastic sediments, mafic to felsic volcanic rocks and pelitic rocks metamorphosed at greenschist facies. These rocks are considered to be the low grade equivalents of the Wutai greenstones (Zhang, 1988; Tian, 1991). Amphibolite from the lower sequence of the Lüliang domain has been dated with the $\mathrm{Sm}-\mathrm{Nd}$ whole-rock isochron method at $2469 \pm 159 \mathrm{Ma}$, interpreted to be a rock-forming age (Zhang, 1988). A number of $\sim 2.0 \mathrm{Ga} \mathrm{Rb}-\mathrm{Sr}$ whole-rock isochron ages have been reported from the low-grade metasedimentary rocks in the upper sequence of the domain (Qiao, 1985). These ages were previously interpreted to be protolith ages, but a preferred interpretation is that they represent mixing ages of metamorphic minerals formed at $\sim 1.8 \mathrm{Ga}$ and partially-reset minerals from the protolith. The metamorphic age of these basement rocks has not been constrained.

Four distinct stages of metamorphism are recognized in garnetiferous amphibolites from the lower sequence. The $\mathrm{M}_{1}$ assemblage plagioclase + hornblende + quartz is preserved as inclusions inside garnet grains. In most cases, the $\mathrm{M}_{1}$ minerals are randomly oriented, but in a few cases, they define the $S_{1}$ foliation by preferred orientation of fine-grained hornblende and elongated quartz. $\mathrm{S}_{1}$ is oblique to the matrix foliation $\mathrm{S}_{2}$. The $\mathrm{M}_{2}$ stage represents the growth of coarse-grained garnet porphyroblasts and matrix mineral assemblage plagioclase + hornblende + quartz \pm clinopyroxene. Garnet is compositionally pyrope-rich and spessartine-poor almandine, without pronounced core to rim compositional variations (Table 2). Matrix plagioclases occur as xenoblasts, without marked core to rim compositional variations. Amphibole occurs as subhedral grains and ranges modally from 30 to $70 \%$. Garnet and minor amounts of clinopyroxene in the $\mathrm{M}_{2}$ assemblage may have been produced from the $\mathrm{M}_{1}$ minerals through the following generalized reactions:

Plagioclase $_{1}+$ hornblende $_{1}$

$\rightarrow$ garnet + plagioclase $_{2}+$ quartz $+\mathrm{H}_{2} \mathrm{O}$

Plagioclase + hornblende + quartz

$\rightarrow$ garnet + clinopyroxene $+\mathrm{H}_{2} \mathrm{O}$

The $\mathrm{M}_{3}$ stage is characterized by the development of plagioclase + cummingtonite symplectites around garnet grains. Symplectic cummingtonites occur as fibrous aggregates intergrown with coronitic plagioclase separating garnet grains from quartz. This texture suggests that the symplectite cummingtonite + plagioclase was produced by the following generalized hydration reaction:

garnet + quartz $+\mathrm{H}_{2} \mathrm{O}$

$\rightarrow$ plagioclase (symplectite)

$$
+ \text { cummingtonite (symplectite) }
$$

The symplectic plagioclase is slightly more calcic than matrix plagioclase, with an An content of 39-49 for symplectic plagioclase and 30-46 for matrix plagioclase (Table 2).

The $\mathrm{M}_{4}$ stage is represented by retrogressive minerals such as chlorite, actinolite and epidote replacing garnet, plagioclase and hornblende grains. These retrogressive minerals may be produced by the following generalized hydration reactions: 
amphibole + plagioclase $+\mathrm{H}_{2} \mathrm{O}$

$\rightarrow$ chlorite + epidote + quartz

plagioclase + garnet $+\mathrm{H}_{2} \mathrm{O} \rightarrow$ epidote + quartz

garnet $+\mathrm{H}_{2} \mathrm{O} \rightarrow$ chlorite + quartz

amphibole $+\mathrm{H}_{2} \mathrm{O}$

$\rightarrow$ chlorite + actinolite + quartz + epidote

THERMOCALC (Appendix A) was used to calculate the $\mathrm{P}-\mathrm{T}$ conditions because this program includes thermodynamic data for cummingtonite that appears in the amphibolites in the Lüliang domain. The $\mathrm{M}_{1}$ conditions were estimated based on the compositions of the plagioclase + hornblende + quartz inclusions and core compositions of inclusion-bearing garnet grains, and the $\mathrm{M}_{2}$ conditions were estimated based on the core compositions of matrix plagioclase, hornblende and garnet porphyroblasts surrounded by matrix plagioclase and quartz. The end-member phases used in the calculations include anorthite, pyrope, almandine, grossular, quartz, tremolite, tschermakite, pargasite and $\mathrm{H}_{2} \mathrm{O}$, which constitute three independent equilibria. These independent equilibria gave average $\mathrm{P}-\mathrm{T}$ estimates of $7.0-7.5 \mathrm{kbar}$ and $500-550^{\circ} \mathrm{C}$ for the $\mathrm{M}_{1}$ assemblage, and 9.0-10.0 kbar and $600-650^{\circ} \mathrm{C}$ for the $\mathrm{M}_{2}$ mineral assemblage, with the standard errors of $\pm 0.6-1.2 \mathrm{kbar}$ and \pm $20-50^{\circ} \mathrm{C}$. The $\mathrm{P}-\mathrm{T}$ calculations of $\mathrm{M}_{3}$ were estimated based on the compositions of symplectic cummingtonite + plagioclase and the rim compositions of garnet. The end-member phases used in the calculations include anorthite, albite, pyrope, almandine, grossular, quartz, tschermakite, grunerite, cummingtonite and $\mathrm{H}_{2} \mathrm{O}$. There are three independent equilibria that can be written between these end-member phases. These equilibria yielded average $\mathrm{P}-\mathrm{T}$ estimates of $6.0-6.5$ kbar and $600-650^{\circ} \mathrm{C}$ for the $\mathrm{M}_{3}$ stage, with the standard errors of $\pm 0.4-1.2$ kbar and $\pm 30-$ $50^{\circ} \mathrm{C}$. The $\mathrm{M}_{4}$ mineral assemblage is typical of greenschist facies with a stability range of $400-$ $500^{\circ} \mathrm{C}$ (cf. Yardley, 1989, pp. 49-51), whereas its pressure cannot be quantitatively estimated because of the lack of diagnostic mineral assemblages. The $\mathrm{P}-\mathrm{T}$ estimates for $\mathrm{M}_{1}-\mathrm{M}_{3}\left(\mathrm{M}_{4}\right)$ define a clockwise $\mathrm{P}-\mathrm{T}$ path for the Lüliang domain (Fig. 6(e)).

\subsection{Zhongtiao domain (ZT)}

The Zhongtiao domain defines the southern margin of the Central Zone (Fig. 2). It consists of TTG gneisses, syn-tectonic granites, and supracrustal rocks including amphibolites, pelitic schists, felsic paragneisses, calc-silicate rocks and conglomeratic rocks metamorphosed at greenschist to amphibolite facies. Sun et al. (1993a) dated the amphibolites by the $\mathrm{Sm}-\mathrm{Nd}$ wholerock isochron method at $2554 \pm 35$ and $2497 \pm$ $51 \mathrm{Ma}$, calc-silicate rocks and tonalitic gneisses by the single-grain zircon $\mathrm{U}-\mathrm{Pb}$ method at $2348 \pm 12$ and $2321 \pm 2 \mathrm{Ma}$, respectively. These ages were interpreted to reflect rock-forming events and thus suggest that the supracrustal assemblage including amphibolites and calc-silicate rocks developed prior to the intrusion of tonalitic plutons (Sun et al., 1993b). A syn-tectonic granite yielded a single-grain zircon $\mathrm{U}-\mathrm{Pb}$ age of $1888 \pm 52 \mathrm{Ma}$, interpreted as the age of peak metamorphism (Sun et al., 1993a).

Textural relations in the garnetiferous pelitic schists indicate three metamorphic stages for the Zhongtiao metamorphic complex (Mei, 1994). $\mathrm{M}_{1}$ is represented by chlorite, muscovite, biotite, garnet (core), plagioclase and quartz, which occur as oriented inclusion trails, defining an early foliation within garnet porphyroblasts. The $\mathbf{M}_{2}$ assemblage is muscovite + biotite + garnet + staurolite + plagioclase + quartz and occurs as porphyroblasts or matrix minerals and defines the regional foliation. The $\mathrm{M}_{3}$ stage is shown by the retrogressive mineral assemblage muscovite + biotite + chlorite \pm garnet $\quad($ rim $) \pm$ plagioclase (rim) \pm quartz. Using the conventional garnet-biotite geothermometer of Ferry and Spear (1978) and garnet-biotitemuscovitequartz geobarometer of Hodges and Crowley (1985), Mei (1994) estimated the $\mathrm{P}-\mathrm{T}$ conditions 
for $\mathrm{M}_{1}$ at $4.0-5.0 \mathrm{kbar}$ and $450-470^{\circ} \mathrm{C}, \mathrm{M}_{2}$ at 5.0-6.0 kbar and $500-600^{\circ} \mathrm{C}$, and $\mathrm{M}_{3}$ at $0.5-1.0$ kbar and $500-550^{\circ} \mathrm{C}$. These $\mathrm{P}-\mathrm{T}$ estimates define a clockwise $\mathrm{P}-\mathrm{T}$ path for the Zhongtiao domain (Fig. 6(f)). Mei (1994) also used the GIBBS program of Spear (1989) to establish a $\mathrm{P}-\mathrm{T}$ path from garnet zonation, which is in agreement with the $\mathrm{P}-\mathrm{T}$ path defined by conventional thermobarometers (Fig. 6(f)).

\subsection{Huaian domain $(H A)$}

The Huaian domain forms the northwestern part of the Central Zone (Fig. 2). The domain comprises high-grade TTG gneisses, granitoids and mafic granulites, with minor amounts of metasedimentary rocks. Recent $\mathrm{U}-\mathrm{Pb}$ and $\mathrm{Sm}-$ Nd dating by Guo et al. (1996), Shen et al. (1994) and Wang et al. (1995) have recognized two main age groups within the Huaian domain at 25002400 and 1900-1800 Ma, which were interpreted as ages of the protolith and the main metamorphic event, respectively.

The metamorphic evolution of the rocks in the Huaian domain has been described by Zhang et al. (1994) and Liu (1995). These authors recognized four metamorphic stages $\left(\mathrm{M}_{1}-\mathrm{M}_{4}\right)$ in both mafic granulites and Al-rich gneisses. In mafic granulites of the Huaian domain, the $\mathrm{M}_{1}$ stage is represented by the assemblage hornblende + plagioclase + quartz \pm magnetite, which occurs as inclusions within orthopyroxene, clinopyroxene and garnet. The $\mathrm{P}-\mathrm{T}$ conditions of $\mathrm{M}_{1}$ were estimated at $7.5-8.5 \mathrm{kbar}$ and $550-650^{\circ} \mathrm{C}$ using the plagioclase-amphibole geothermobarometers (Plyusnina, 1982; Holland and Blundy, 1994). The $\mathbf{M}_{2}$ stage is represented by orthopyroxene + clinopyroxene + garnet + plagioclase + quartz. In some cases, orthopyroxene, clinopyroxene and garnet contain the $\mathbf{M}_{1}$ assemblage hornblende + plagioclase + quartz \pm magnetite, suggesting that they may be produced through the following generalized reactions:

\section{hornblende + quartz}

$\rightarrow$ orthopyroxene + clinopyroxene + plagioclase

$$
+\mathrm{H}_{2} \mathrm{O}
$$

hornblende + plagioclase + quartz + magnetite

$\rightarrow$ clinopyroxene + garnet $+\mathrm{H}_{2} \mathrm{O}+\mathrm{O}_{2}$

The $\mathrm{P}-\mathrm{T}$ conditions of $\mathrm{M}_{2}$ were estimated at $9.0-11.0 \mathrm{kbar}$ and $800-850^{\circ} \mathrm{C}$ using the garnetclinopyroxene geothermometers (Ellis and Green, 1979; Ganguly et al., 1988) and garnet-orthopyroxene-plagioclase-quartz (Newton and Perkins, 1982), garnet-clinopyroxene-plagioclase-quartz (Newton and Perkins, 1982), garnet-clinopyroxene-orthopyroxene-plagioclase-quartz (Paria et al., 1988) and orthopyroxene-garnet geobarometers (Harley, 1984). The $\mathbf{M}_{3}$ stage is represented by the assemblage orthopyroxene + plagioclase + clinopyroxene \pm magnetite which occur as symplectites or coronas surrounding embayed garnet grains. They may have been produced through reactions (1), (2) and (3). The same geothennobarometers used for the $\mathrm{M}_{2}$ stage yielded 6.5-7.5 kbar and $700-750^{\circ} \mathrm{C}$ for the $\mathrm{M}_{3}$ stage, based on the rim compositions of garnet and symplectic or coronitic plagioclase, orthopyroxene and clinopyroxene. The $\mathrm{M}_{4}$ stage is represented by the mineral assemblage cummingtonite + plagioclase, which occur as retrogressive rims surrounding orthopyroxene and clinopyroxene grains. The $\mathrm{P}-$ $\mathrm{T}$ conditions of this stage cannot be quantitatively estimated because of the lack of suitable geothermometers and geobarometers, but in most cases, the transition from pyroxene $\left(\mathrm{M}_{3}\right)$ to cummingtonite $\left(\mathrm{M}_{4}\right)$ indicates a retrogressive metamorphic process (Barker, 1989, p. 61) Thus, the mineral assemblages and available $\mathrm{P}-\mathrm{T}$ estimates for the mafic granulites define a clockwise $\mathrm{P}-\mathrm{T}$ path for the Huaian domain (Fig. 6(g)).

In the Al-rich gneisses, the $\mathrm{M}_{1}$ stage is represented by the assemblages kyanite + garnet $($ core $)+$ biotite + plagioclase + quartz, which are present as inclusions within the garnet grains and define an early foliation $\left(\mathrm{S}_{1}\right)$. The $\mathrm{P}-\mathrm{T}$ conditions of $\mathrm{M}_{1}$ were estimated at $600-670^{\circ} \mathrm{C}$ and pressures of 7.5-8.5 kbar using the garnet-biotite geothermometer of Ferry and Spear (1978) and the garnet-plagioclase-kyanite-quartz geobarometer of Koziol and Newton (1988). The $\mathbf{M}_{2}$ stage is defined by the mineral assemblages sillimanite + garnet (rim) + biotite + plagioclase + quartz, which occur as a matrix and define the regional 
foliation $\left(\mathrm{S}_{2}\right)$. The $\mathrm{M}_{2}$ assemblage differs from the $\mathrm{M}_{1}$ assemblage only in the absence of kyanite and the presence of sillimanite. The reactions for the transition from $M_{1}$ to $M_{2}$ remains unknown because of the lack of clear reaction relations. The $\mathrm{P}-\mathrm{T}$ conditions of $\mathrm{M}_{2}$ have been estimated at $9.5-11.0 \mathrm{kbar}$ and $800-850^{\circ} \mathrm{C}$ with the garnet-biotite geothermometer of Ferry and Spear (1978) and the garnet-plagioclase-sillimanite-quartz geobarometer of Koziol and Newton (1988). The $\mathrm{M}_{3}$ stage is shown by: (1) cordierite coronas around garnet, (2) cordierite + orthopyroxene symplectites on garnet grains, (3) cordierite + spinel coronas around garnet grains, and (4) spinel + K-feldspar + quartz symplectite matrix biotite (Liu, 1995). These coronitic and symplectic textures suggest the following generalized reactions for the transition from $\mathrm{M}_{2}$ to $\mathrm{M}_{3}$ :

garnet + quartz $\rightarrow$ orthopyroxene + cordierite

garnet + sillimanite + quartz $\rightarrow$ cordierite

garnet + sillimanite $\rightarrow$ spinel + cordierite

biotite + sillimanite

$\rightarrow$ spinel + K-feldspar + quartz $+\mathrm{H}_{2} \mathrm{O}$

Reactions (17), (18) and (19) are considered to indicate a decompressional process (Hensen and Green, 1972; Vielzeuf, 1983). The P-T conditions of $\mathrm{M}_{3}$ were estimated at $650-750^{\circ} \mathrm{C}$ and $5.0-6.5$ kbar using the garnet-cordierite thermometer of Aranovich and Kosyakova (1983) and the garnet-cordierite-sillimanite-quartz barometers of Thompson (1976) and Aranovich and Kosyakova (1983). The $\mathrm{M}_{4}$ stage is represented by the retrogressive mineral assemblage of biotite $+\mathrm{K}$ feldspar + magnetite surrounding garnet, or fine-grained muscovite + quartz surrounding sillimanite or cordierite. The possible reactions for the transition from $\mathrm{M}_{3}$ to $\mathrm{M}_{4}$ may be involved in fluids containing $\mathrm{K}_{2} \mathrm{O}$ and $\mathrm{N}_{2} \mathrm{O}$ (Lu et al., 1992). The metamorphic temperature of this stage was semi-quantitatively estimated at $550-620^{\circ} \mathrm{C}$ using the garnet-biotite thermometer of Ferry and Spear (1978), but the pressure cannot be quantitatively estimated. Therefore, the $\mathrm{P}-\mathrm{T}$ estimates from Al-rich gneisses also define a clockwise $\mathrm{P}-\mathrm{T}$ path for the Huaian domain (Fig. 6(h)). Similar $\mathrm{P}-\mathrm{T}$ paths have been inferred by Zhang et al. (1994).

\section{7. $H P G$ domain}

HPG from the Central Zone of the North China Craton are referred to as those granulite facies metabasic rocks which contain the main (peak) mineral assemblage clinopyroxene + plagioclase + garnet + quartz. They are distinguished from eclogites by the presence of plagioclase and from medium-pressure granulites by the lack of orthopyroxene in the main mineral assemblage. Recent geological investigations reveal that the high-pressure granulites within the Central Zone occur along a northeast-southwest trending zone that extends from the Hengshan area (Wang et al., 1991), through the Huaian (Zhai et al., 1992, 1995; Guo et al., 1996) and Xuanhua (Wang et al., 1994), into the northern Hebei Province (Li et al., 1998), a distance of $\sim 500 \mathrm{~km}$ (Fig. 2). In the field, the high-pressure granulites are restricted to enclaves, boudins and sheets, ranging from 0.1 to $2 \mathrm{~m}$ in width and 0.1 to $50 \mathrm{~m}$ in length, within heterogeneous, migmatitic veined and deformed upper amphibolite to granulite facies TTG gneisses. The protoliths of these high-pressure granulites remains unknown. They may be metamorphosed basalts or basic intrusives, including gabbro and dolerite dykes; the latter appears to be the most likely interpretation (Kröner, personal communication, 1999). Sm-Nd whole-rock isotopic analyses from six high-pressure granulite samples gave a grouped age of $2647 \pm 115 \mathrm{Ma}$ (Guo et al., 1996), which was interpreted as the protolith age. $\mathrm{Sm}-\mathrm{Nd}$ isotopic analyses on the metamorphic minerals garnet, clinopyroxene, orthopyroxene, hornblende and the whole rock yielded an isochron age of $1827 \pm 18 \mathrm{Ma}$ (Guo et al., 1996). Zircons separated from the above six $\mathrm{Sm}-\mathrm{Nd}$ samples also yielded an upper intercept age of $1833 \pm 24 \mathrm{Ma}$ (Guo et al., 1996). These data are considered to represent the age of the main high-pressure metamorphic event.

Petrographic evidence from the high-pressure granulites in the Hengshan domain indicates four distinct metamorphic assemblages. The early pro- 
grade assemblage $\left(M_{1}\right)$ is represented by quartz and rutile inclusions within the cores of garnet porphyroblasts, and omphacite pseudomorphs that are indicated by clinopyroxene + sodic plagioclase $\left(\mathrm{An}_{10-20}\right)$ symplectic intergrowths of which the exsolution-like sodic plagioclases make up to 30-40 vol.\% (Fig. 8(a)). Similar textures have also been observed in many other high-pressure granulites and retrograded eclogites, and are thought to indicate the replacement of omphacite by plagioclase and clinopyroxene through the following solid-solid reactions during the transition from eclogite facies to high-pressure facies (Heinrich, 1982; Rubie, 1990; Smelov and Beryozkin, 1993; Möller, 1998):

omphacite + quartz

$\rightarrow$ sodic plagioclase + clinopyroxene
The peak assemblage $\left(\mathrm{M}_{2}\right)$ consists of clinopyroxene + garnet + sodic plagioclase + quartz \pm hornblende. Garnet show pronounced compositional variations, with a decrease in almandine and pyrope and an increase in grossular, spessartine and $\mathrm{X}_{\mathrm{Fe}}[=\mathrm{Fe} /(\mathrm{Fe}+\mathrm{Mg})]$ from rim to core (Table 3 and Fig. 4(c)). The cores of large subhedral grains are compositionally homogeneous with relatively flat profiles (Fig. 4(c)), which we interpret as having developed during peak metamorphism. The outermost rims, ca. $0.5 \mathrm{~mm}$ wide, have low grossular and high almandine contents (Fig. 4(c)), which reflect resetting by diffusion and/or net-transfer reactions during post-peak decompression and cooling. Plagioclase also shows a distinct compositional zoning, varying in composition from $\mathrm{An}_{12}$ to $\mathrm{An}_{46}$, with an oligoclase core and an andesine rim (Table 3 and
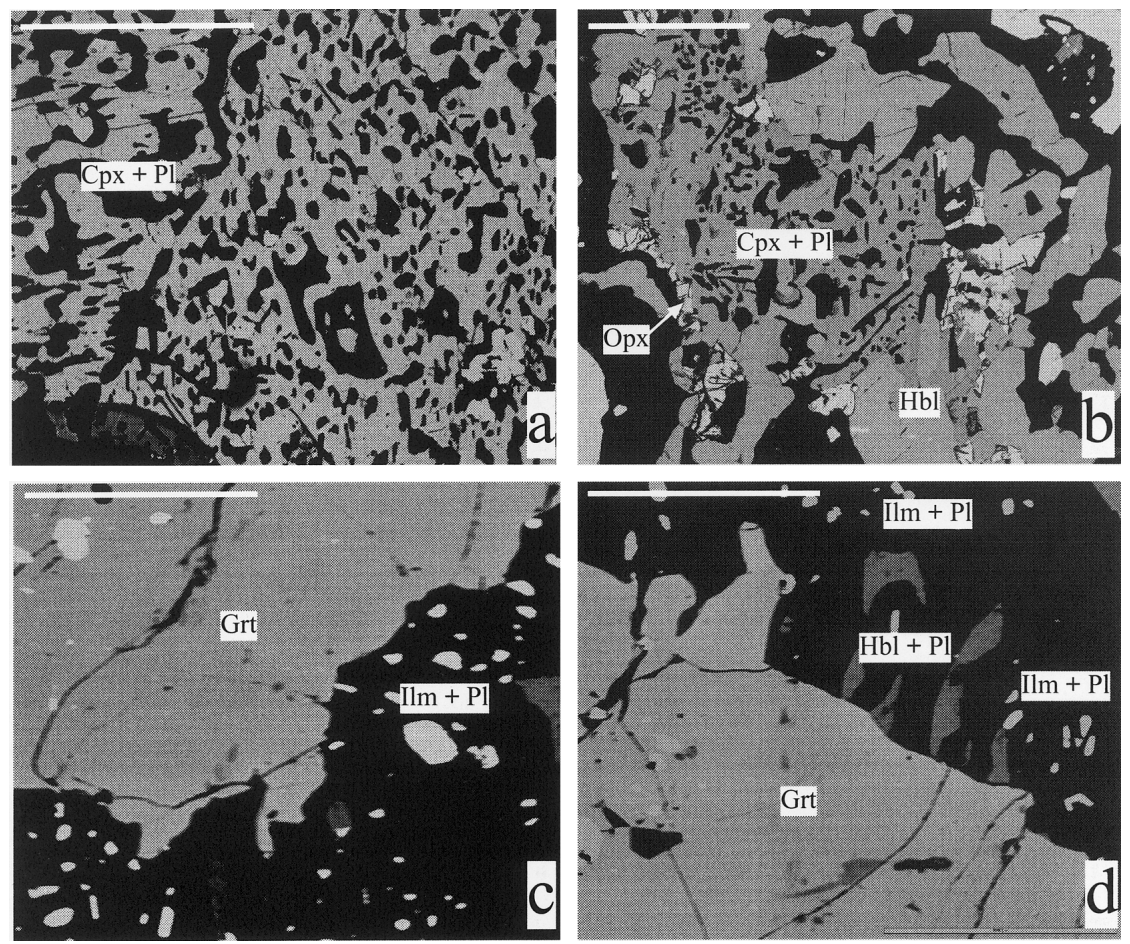

Fig. 8. Back-scattered electron images showing representative metamorphic reaction textures of high-pressure granulites. Scale bar: $1 \mathrm{~mm}$. (a) Omphacite pseudomorph indicated by sodic plagioclase + clinopyroxene symplectic intergrowths. (b) Clinopyroxene + sodic plagioclase symplectite rimmed by fine-grained orthopyroxenes. (c) Plagioclase + ilmenite symplectite around garnet. (d) Plagioclase + hornblende symplectite separating plagioclase + ilmenite symplectite from garnet. Mineral symbols are after Kreze (1983). 
Fig. 4(d)). Clinopyroxenes show irregular zoning patterns, especially with respect to $\mathrm{Ca}, \mathrm{Mg}$ and $\mathrm{Fe}$, and their $\mathrm{Al}$ contents increase slightly from rim to core, whereas $\mathrm{Na}$ contents are nearly uniform (Table 3 and Fig. 4(e)). There is a marked difference in compositions between the core and rim of hornblende. The core is higher in $\mathrm{SiO}_{2}$ and $\mathrm{MgO}$ but lower in $\mathrm{Al}_{2} \mathrm{O}_{3}$ than the rim (Table 3 and Fig. 4(f)). These core to rim composition variations could reflect the early highpressure equilibration and partial re-equilibration during post-peak thermal events.

The $M_{3}$ stage represents the development of orthopyroxene + clinopyroxene + plagioclase symplectites and coronas surrounding embayed garnet grains. The symplectic texture consists of intergrowths of fine-grained, worm-like orthopyroxene + plagioclase \pm magnetite around embayed garnet grains. The corona textures consist of very elongate plagioclase, clinopyroxene and/ or orthopyroxene, separating garnet and quartz, where plagioclase is always present adjacent to garnet, and the clinopyroxene and/or orthopyroxene mantles quartz (Fig. 8(b)). In some places, garnet is only surrounded by plagioclase + ilmenite symplectites, without symplectic orthopyroxene (Fig. 8(c)). The symplectic or coronitic orthopyroxene, plagioclase and clinopyroxene may be produced by a combination of decompressional reactions (1), (2) and (3); the symplectite plagioclase + ilmenite may be produced through the following generalized reaction:

garnet + rutile $\rightarrow$ ilmenite + plagioclase + quartz

Symplectic or coronitic plagioclase are generally labradorite to bytownite, more calcic than matrix plagioclase (Table 3), without a pronounced compositional zoning (Fig. 4(g)). Symplectic or coronitic orthopyroxene is also compositionally homogeneous with a relatively flat compositional profile (Fig. 4(h)).

The $\mathrm{M}_{4}$ is represented by hornblende + plagioclase symplectites which occur as worm-like intergrowths adjacent to garnet. They are petrographically similar to the symplectites in the medium mafic granulites discussed in the Hengshan, Fuping and Huaian domains.

The $\mathrm{P}-\mathrm{T}$ conditions of the early prograde assemblage $\left(\mathrm{M}_{1}\right)$ cannot be quantitatively estimated because of the absence of representative high-pressure minerals (e.g. omphacite). The THERMOCALC program yielded $\mathrm{P}-\mathrm{T}$ conditions of $13.4-15.5 \pm 1.5 \mathrm{kbar}$ and $770-840 \pm$ $50^{\circ} \mathrm{C}$ for the peak mineral assemblage $\left(\mathrm{M}_{2}\right)$, based on the core compositions of garnet, matrix clinopyroxene, hornblende and plagioclase. The $\mathrm{P}-\mathrm{T}$ conditions of orthopyroxene + plagioclase \pm magnetite symplectite and clinopyroxene + orthopyroxene + plagioclase corona $\left(\mathrm{M}_{3}\right)$ were estimated at ca. 6.5-8.0 $\pm 1.0 \mathrm{kbar}$ and $750-830 \pm 40^{\circ} \mathrm{C}$, and hornblende + plagioclase symplectite $\left(\mathrm{M}_{4}\right)$ at $4.5-6.0 \pm 1.2 \mathrm{kbar}$ and $680-$ $790 \pm 60^{\circ} \mathrm{C}$. The combination of petrographic textures, mineral compositions and thermobarometric data defines a near-isothermal decompressional clockwise $\mathrm{P}-\mathrm{T}$ path for the Hengshan high-pressure granulites (Fig. 6(i)). A similar $\mathrm{P}_{-}$ $\mathrm{T}$ path has been established for the high-pressure granulites in the Huaian domain (Fig. 6(i); Zhai et al., 1992).

\subsection{Northern Hebei domain (NH)}

The Northern Hebei domain, previously named the Hongqiyingzi Complex (Zhang, 1990), is located in the northern part of the Central Zone (Fig. 2). The domain comprises felsic gneisses, amphibolites, marbles and mica schists in low amphibolite facies, locally at upper amphibolite to granulite facies (Zhang, 1990). Garnetiferous mica schists display three mineral assemblages: garnet (core) + staurolite + kyanite + muscovite + biotite + plagioclase + quartz $\left(\mathrm{M}_{1}\right)$, garnet + sillimanite + muscovite + biotite + plagioclase + quartz $\left(\mathrm{M}_{2}\right)$, and chlorite + muscovite $\left(M_{3}\right)$ (Zhang, 1990). Except for garnet, all other $\mathrm{M}_{1}$ minerals occur as mineral inclusions within garnet porphyroblasts; the $\mathrm{M}_{2}$ minerals are present as porphyroblasts (garnet) and matrix, and the $\mathrm{M}_{3}$ minerals occur as retrogressive rims surrounding garnet grains. The $\mathrm{P}-$ $\mathrm{T}$ evolution of these three assemblages can be 


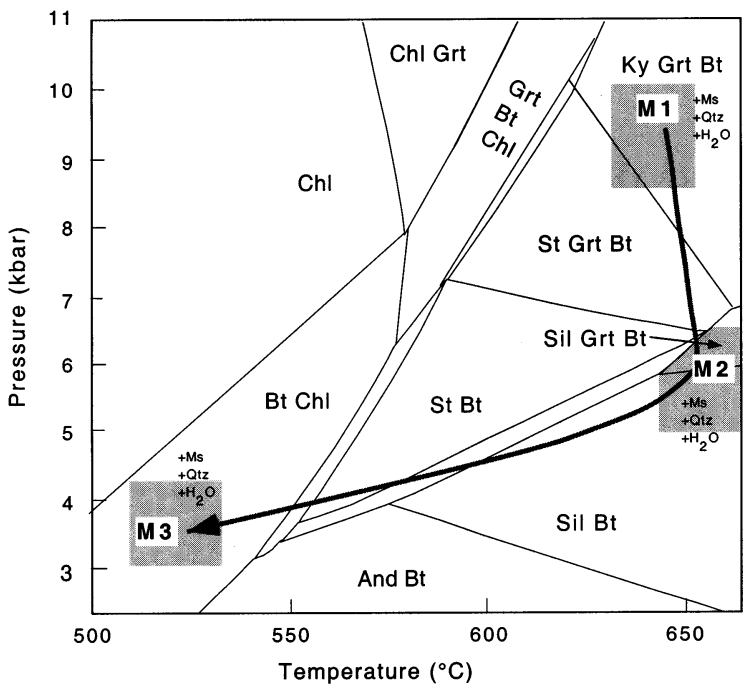

Fig. 9. $\mathrm{P}-\mathrm{T}$ pseudosection for a portion of the KFMASH system (after Powell et al., 1998) showing the qualitative $\mathrm{P}-\mathrm{T}$ path of the northern Hebei metapelitic schists constrained by the sequence of mineral assemblages. Mineral symbols are after Kreze (1983).

qualitatively defined by a $\mathrm{P}-\mathrm{T}$ pseudosection (Fig. 9) of the KFMASH grid of Powell et al. (1998). This KFMASH ( + muscovite + quartz + $\mathrm{H}_{2} \mathrm{O}$ ) grid shows a prograde sequence of mineral assemblages similar to those observed in the pelitic schists from the Northern Hebei domain. For example, the staurolite + garnet + biotite and kyanite + garnet + biotite fields can be used to show the $M_{1}$ assemblage; the sillimanite + garnet + biotite and sillimanite + biotite fields can be used to show the $\mathbf{M}_{2}$ assemblage; the chlorite + biotite field can be used to show the $\mathrm{M}_{3}$ assemblage. In each field muscovite + quartz $+\mathrm{H}_{2} \mathrm{O}$ are assumed present. As seen in Fig. 9, the equilibrium pressures of the staurolite + garnet + biotite and kyanite + garnet + biotite fields are higher than that of the sillimanite + garnet + biotite and sillimanite + biotite fields, and thus, the transition from $\mathrm{M}_{1}$ to $\mathrm{M}_{2}$ represents a decompressional process. The chlorite + biotite field has a wide $\mathrm{P}-\mathrm{T}$ scope, but in most cases, retrogression of garnet into chlorite and/or biotite occurs in association with declining $\mathrm{P}-\mathrm{T}$ conditions (Barker, 1989, pp. 6061 ), and thus, the $M_{3}$ assemblage of chlorite + biotite + muscovite is assumed present in an area with $\mathrm{P}-\mathrm{T}$ conditions lower than those of the $\mathrm{M}_{2}$ assemblage. Taken together, a clockwise $\mathrm{P}-\mathrm{T}$ path has been qualitatively inferred (Fig. 9).

The garnetiferous amphibolites from the Northern Hebei domain exhibit two distinct mineral assemblages. The $\mathbf{M}_{1}$ assemblage is garnet + amphibolite + plagioclase + quartz, representing the growth of porphyroblasts and matrix minerals; the $\mathbf{M}_{2}$ assemblage is represented by plagioclase + hornblende symplectites around garnet grains. The $\mathrm{M}_{1} \mathrm{P}-\mathrm{T}$ conditions were estimated at $9.0-10.5 \mathrm{kbar}$ and $600-650^{\circ} \mathrm{C}$ with the garnet-amphibole thermometer of Graham and Powell (1984) and the garnet + amphibolite + plagioclase + quartz barometry of Kohn and Spear (1990). The P-T conditions of plagioclase + hornblende symplectites cannot be estimated because of the lack of analytical data, but in the majority of cases the development of hornblende + plagioclase symplectites surrounding garnet grains is considered to the direct result of decompression (cf. Barker, 1989, pp. 55-56). Therefore, a clockwise $\mathrm{P}-\mathrm{T}$ path can be inferred from the amphibolites for the Northern Hebei domain.

\section{Tectonic implications}

As shown in Fig. 6, the metamorphic domains in the Central Zone of the North China craton, regardless of their protolith age, metamorphic grade and composition, are all characterized by clockwise $\mathrm{P}-\mathrm{T}$ paths. Some errors may exist in $\mathrm{P}-\mathrm{T}$ estimates because of the use of inconsistent thermobarometry for some domains, but the similarity in metamorphic evolution among these domains is clearly not an artefact of thermobarometry since the inferred $\mathrm{P}-\mathrm{T}$ paths are constrained not only by $\mathrm{P}-\mathrm{T}$ estimates, but also by metamorphic reaction textures. Metamorphic textures from mafic granulites, amphibolites and pelitic rocks in the Central Zone indicate a nearly isothermal decompression process $\left(\mathrm{M}_{2}\right)$ 
following the peak metamorphism $\left(\mathrm{M}_{1}\right)$ and then decompressional cooling process $\left(\mathrm{M}_{3}\right)$. The nearly isothermal decompression $\left(\mathrm{M}_{2}\right)$ textures are represented by worm-like orthopyroxene/ clinopyroxene + plagioclase symplectites or coronas in mafic granulites, hornblende/cummingtonite + plagioclase symplectites in amphibolites and cordierite \pm orthopyroxene \pm spinel symplectites in high-grade pelitic gneisses. The decompressional cooling $\left(\mathrm{M}_{3}\right)$ textures are shown by hornblende + plagioclase symplectic coronas in mafic granulites, chlorite + epidote + mica retrogressive rims around garnet or hornblende grains in amphibolites, and biotite $+\mathrm{K}$ feldspar + magnetite replacing garnet, cordierite, sillimanite in pelitic rocks. These textural relations define a clockwise $\mathrm{P}-\mathrm{T}$ evolution.

The estimated clockwise $\mathrm{P}-\mathrm{T}$ paths for different domains of the Central Zone is compatible with a tectonothermal process characterized by initial crustal thickening, subsequent nearisothermal exhumation and final cooling, a sequence of tectonothermal events typical of continent-conti-nent collisional environments (England and Thompson, 1984; Thompson and England, 1984). Considering the spatial and temporal relationships between basement rocks in the Central Zone and Eastern and Western Blocks of the North China Craton, it is believed that the sequence of tectonic events inferred from the $\mathrm{P}-\mathrm{T}$ evolution of the basement rocks in the Central Zone was related to the collision between the Eastern and Western Blocks. Geochronological data invariably indicate that this collisional event occurred at $\sim 1.8 \mathrm{Ga}(\mathrm{Wu}$ and Zhong, 1998).

Based on this study and previous work (Zhao et al., 1998, 1999a,b; Zhao et al., in press), it was proposed that in the late Archean to Paleoproterozoic, the Eastern and Western Blocks in the North China Craton existed as two separate continental blocks. The Eastern Block had an active-type continental margin on which intracontinental arcs and intra-arc basins developed and subsequently became the Central Zone, whereas the Western Block had a passive-type continental margin on which stable continental margin sediments were deposited and subsequently became the khondalites present along the eastern margin of the Western Block (Zhao et al., 1999a). Separating the two continental blocks was an old ocean, the floor of which was subducted beneath the western margin of the Eastern Block (Fig. 10(a)). By $\sim 1.8 \mathrm{Ga}$, the old ocean between the two blocks disappeared by complete subduction, and the collision between the Eastern and Western Blocks occurred (Fig. 10(b,c)). During the collision, the Paleoproterozoic sedimentary formation on the passive continental margin of the Western Block was obducted over the active-type continental margin of the Eastern Block (Fig. 10(b)), because of its relatively low density. This can explain the tectonic relationship that some Paleoproterozoic khondalites were thrusted over the granulite facies terrains (e.g. Huaian, HPG and Fuping domains) in the Central Zone. The collision caused the crust of the Central Zone to double in thickness and to be metamorphosed $\left(\mathrm{M}_{1}\right)$ in medium- to high-pressure granulite facies (e.g. the Hengshan, Huaian, Fuping, Taihua and HPG domains) in the lower crust and greenschist to amphibolite facies, (e.g. the $\mathrm{Wu}$ tai, Lüliang, Zhongtiao, Dengfeng, Zanhuang and Northern Hebei domains) in the upper crust. Following peak metamorphism, the thickened crust underwent exhumation driven by crustal isostatic compensation and accompanied decompression metamorphism $\left(\mathrm{M}_{2}\right)$ which resulted in the development of widespread symplectic textures in the rocks. Finally, retrogressive cooling, accompanied by hydration $\left(\mathrm{M}_{3}\right)$ occurred in the metamorphic crust when exhumation ceased. The mineral reaction textural relations and $\mathrm{P}-\mathrm{T}$ paths of the basement rocks in the Central Zone record the tectonothermal history of collision between the Eastern and Western Blocks which resulted in final assembly of the North China Craton. The clockwise $\mathrm{P}-\mathrm{T}$ paths of some Archean mafic granulites and TTG gneisses in the Western Block adjacent to the boundary with the Central Zone reflect reworking of the Archean basement rocks during the Paleoproterozoic collisional event. 

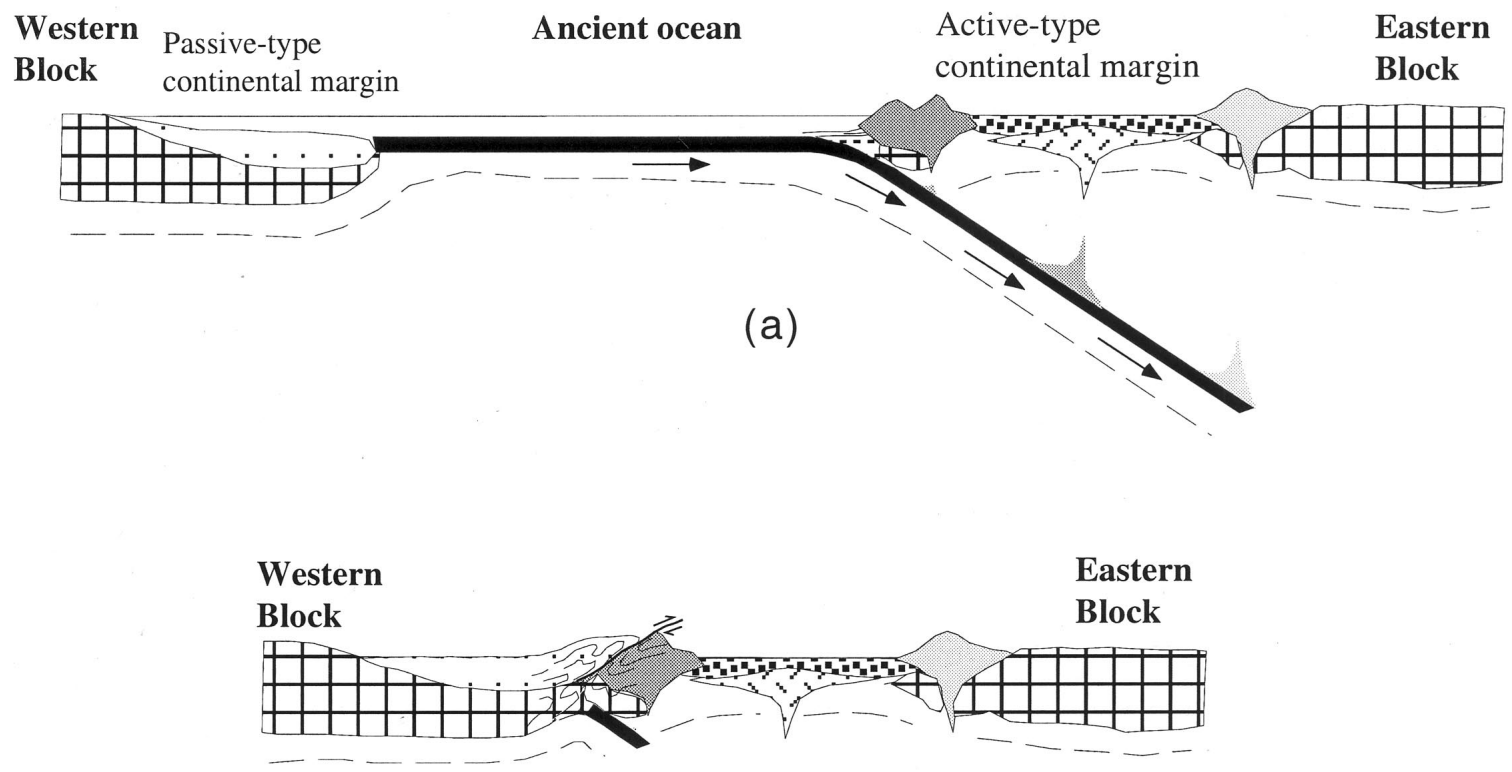

(b)

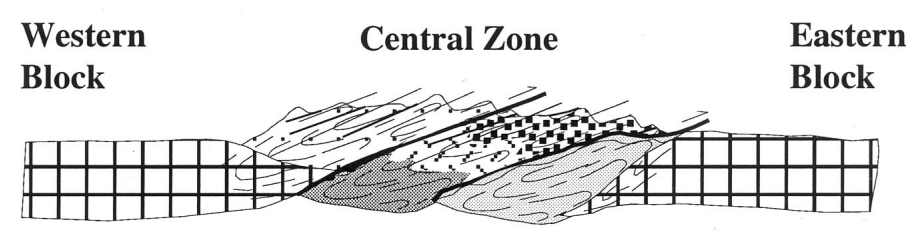

(c)

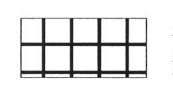

Archean continental basement in the Eastern and Western Blocks

Late Archean to Paleoproterozoic remnant magmatic arc formation

Late Archean to Paleoproterozoic sedimentary formation in intra-arc basins

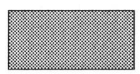

Late Archean to Paleoproterozoic continental magmatic arc formation

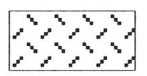

Late Archean to Paleoproterozoic volcanic formation in intra-arc basins

... Paleoproterozoic sedimentary

. . . f formation on passive continental margins

Fig. 10. Schematic sections showing the pre- and syn-collisional geometry of the Eastern and Western Blocks and the Central Zone in the North China Craton. 


\section{Acknowledgements}

We thank F. Mengel and S. Harley for their critical but constructive comments that led to substantial changes and improvements in this manuscript. We also appreciate numerous discussions with A. Kröner, K.Y. Wang, M.G. Zhai and S.W. Liu on the North China Craton. The work was supported by an ARC Large Grant (No. A39532446) to S.A. Wilde and P.A. Cawood and a RGC Grant (HKU 7300/99P) to M. Sun. This is Tectonics Special Research Centre Publication No. 88.

\section{Appendix A. TWQ and THERMOCALC programs}

Of a number of thermobarometric techniques based on internally consistent thermodynamic database, THERMOCALC (Powell and Holland, 1994; Holland and Powell, 1998) and TWQ (Berman, 1991) have been the most widely used. Both programs calculate pressures and temperatures from the intersections of two or more endmember reactions in $\mathrm{P}-\mathrm{T}$ space using one set of internally consistent thermodynamic data. A major difference between THERMOCALC and TWQ is that the former calculates average pres-sures and temperatures based on an independent set of equilibria, whereas the latter uses all possible equilibria to compute pressures and temperatures (Berman, 1991). In addition, the THERMOCALC program allows the likely uncertainties in the results of $\mathrm{P}-\mathrm{T}$ calculations to be estimated, whereas the TWQ program does not enable the reliable calculation of uncertainties to be performed. Generally, a maximum 'aggregate uncertainty' of $\pm 1.0 \mathrm{kbar}$ and $\pm 50^{\circ} \mathrm{C}$ is adopted in TWQ calculations, based on the suggestions of Essene (1989) for conventional thermobarometry. One of the advantages of the TWQ technique is that the equilibration state of a sample can be assessed by comparing the intersection positions of all independent equilibria for a given assemblage. A good convergence is consistent with the inference of equilibrium, whereas divergence suggests that one or more phases were erroneously included in the assemblage.
In present study, average $\mathrm{P}-\mathrm{T}$ calculations by THERMOCALC followed the method of Powell and Holland (1994), using version 2.75 of THERMOCALC and an updated and expanded version of the internally consistent thermodynamic dataset (Holland and Powell, 1998). Mineral activities were calculated for pyroxenes following Holland and Powell (1998), using an ideal two-site mixing model; for garnet following Berman (1990), using the ternary mixing model; for plagioclase following Holland and Powell (1992), using Darken's quadratic formulism; and for hornblende following Holland and Blundy (1994), using a non-ideal mixing model. Quartz was assumed to be pure.

In TWQ, average $\mathrm{P}-\mathrm{T}$ calculations followed the method of Berman (1991), using versions 2.02 or 1.02 (for amphibole-bearing assemblages) of TWQ and the internally consistent thermodynamic datasets of Berman (1988), Berman et al. (1995) and Berman and Aranovich (1996) for end-member phases. Activity-composition relations for garnet, plagioclase, biotite and amphibole were computed using the models of Berman (1990), Fuhrman and Lindsley (1988), McMullin et al. (1991) and Mäder et al. (1994), respectively. Ideal solution models were assumed for ilmenite, orthopyroxene and clinopyroxene. Quartz was assumed to be pure.

\section{References}

Aranovich, L.Y., Kosyakova, N.A., 1983. The cordierite-garnet-sillimanite-quartz equilibrium: experiments and applications. In: Saxena, S.K. (Ed.), Advances in Physical Geochemistry, Kinetics and Equilibrium in Mineral Reactions. Springer-Verlag, New York, pp. 172-198.

Bai, J., 1986. Crustal evolution of Wutaishan area. In: Bai, J. (Ed.), The early Precambrian Geology of Wutaishan. Tianjin Science and Technology Press, Tianjin, China, pp. 373-383 in Chinese with English abstract.

Bai, J., Dai, F.Y., 1998. Archean crust of China. In: Ma, X.Y., Bai, J. (Eds.), Precambrian Crust Evolution of China. Springer-Geological Publishing House, Beijing, pp. 15-86.

Bai, J., Wang, R.Z., Guo, J.J., 1992. The Major Geologic Events of Early Precambrian and Their Dating in Wutaishan Region. Geological Publishing House, Beijing, pp. 1-55 in Chinese with English abstract.

Barker, A.J., 1989. Introduction to Metamorphic Textures and Microstructures. Blackie, Glasgow, pp. 60-62.

Berman, R.G., 1988. Internally consistent thermodynamic data for minerals in the system $\mathrm{N}_{2} \mathrm{O}-\mathrm{K}_{2} \mathrm{O}-\mathrm{CaO}-\mathrm{MgO}-$ 
$\mathrm{FeO}-\mathrm{Fe}_{2} \mathrm{O}_{3}-\mathrm{Al}_{2} \mathrm{O}_{3}-\mathrm{SiO}_{2}-\mathrm{TiO}_{2}-\mathrm{H}_{2} \mathrm{O}-\mathrm{CO}_{2}$. J. Petrol. 29, $445-522$.

Berman, R.G., 1990. Mixing properties of $\mathrm{Ca}-\mathrm{Mg}-\mathrm{Fe}-\mathrm{Mn}$ garnets. Am. Miner. 75, 328-344.

Berman, R.G., 1991. Thermobarometry using multi-equilibrium calculations: a new technique, with petrological applications. Can. Miner. 29, 833-855.

Berman, R.G., Aranovich, L.Y., 1996. Optimized standard state and mixing properties of minerals: I. Model calibration for olivine, orthopyroxene, cordierite, garnet, and ilmenite in the system $\mathrm{FeO}-\mathrm{MgO}-\mathrm{CaO}-\mathrm{Al}_{2} \mathrm{O}_{3}-\mathrm{TiO}_{2}-$ $\mathrm{SiO}_{2}$. Contrib. Mineral. Petrol. 126, 1-24.

Berman, R.G., Aranovich, L.Y., Pattison, D.R.M., 1995. Reassessment of the garnet-clinopyroxene $\mathrm{Fe}-\mathrm{Mg}$ exchange thermometer: II. Thermodynamic analysis. Contrib. Mineral. Petrol. 119, 30-42.

Bohlen, S.K., 1987. Pressure-temperature-time paths and tectonic model for the evolution of granulites. J. Geol. 95, $617-632$.

Bohlen, S.K., 1991. On the formation of granulites. J. Metamorph. Geol. 9, 223-229.

Brown, M., 1993. P-T $-\mathrm{t}$ evolution of orogenic belts and the causes of regional metamorphism. J. Geol. Soc. Lond. 150, 227-241.

Cawood, P., Wilde, S.A., Wang, K.Y., Nemchin, A., 1998. Integrated geochronology and field constraints on subdivision of the Precambrian in China: Data from the Wutaishan. Abstract of the 9th International Conference on Geochronology, Cosmochronology and Isotope Geology, Beijing. Chin. Sci. Bull. 43, 17.

Chamberlain, C.P., Karabinos, P., 1987. Influence of deformation on pressure-temperature paths of metamorphism. Geology 15, 42-44.

Chen, H.S., Zhou, H.F., Li, H.Q., Fluang, B., Ye, B.D., Li, Z.C., Bai, Y.B., Lin, S.L., 1980. Geochronological investigation of the late Archean iron-bearing metamorphic rocks from central Henan, China. China Acad. Geol. Sci. Bull. 8, 88-102 in Chinese.

Chen, N.S., Wang, R.J., Shan, W.Y., Zhong, Z.Q., 1994. Isobaric cooling $\mathrm{P}-\mathrm{T}-\mathrm{t}$ path of the western section of the Nflyun Complex and its tectonic implications. Sci. Geol. Sin. 29, 354-364 in Chinese with English abstract.

Cui, W.Y., Wang, C.Q., Wang, S.G., 1991. Geochemistry and metamorphic $\mathrm{P}-\mathrm{T}-\mathrm{t}$ path of the Jianping Complex in the western Liaoning Province. Acta Petrol. Sin. 7, 13-26 in Chinese with English abstract.

Deer, W.A., Howie, R.A., Zussman, J., 1992. The RockForming Minerals, 2nd edition. Longman Group, Harlow, pp. $548-551$.

Ellis, D., Green, D.H., 1979. An experimental study of the effect of $\mathrm{Ca}$ upon garnet-clinopyroxene $\mathrm{Fe}-\mathrm{Mg}$ exchange equilibria. Contrib. Mineral. Petrol. 71, 13-22.

England, P.C., Thompson, A.B., 1984. Pressure-temperaturetime paths of regional metamorphism, I. Heat transfer during the evolution of regions of thickened continental crust. J. Petrol. 25, 894-928.
Essene, E.J., 1989. The current status of thermobarometry in metamorphic rocks. In: Daly, J.S., Cliff, R.A., Yardley, B.W.D. (Eds.), Evolution of Metamorphic Belts. Geol. Soc. Spec. Pub. 43, 1-44.

Ferry, J.M., Spear, S.F., 1978. Experimental calibration of partition of $\mathrm{Fe}$ and $\mathrm{Mg}$ between biotite and garnet. Contrib. Mineral. Petrol. 66, 113-117.

Fuhrman, M.L., Lindsley, D.H., 1988. Ternary-felspar modelling and thermometry. Am. Miner. 73, 201-216.

Ganguly, J., Bhattacharya, S., Chakraborty, S., 1988. Convolution effect in the determination of compositional profiles and diffusion co-efficients by microprobe step scans. Am. Mineral. 73, 901-909.

Ge, W.C., Sun, D.Y., Wu, F.Y., Lin, Q., 1994. P-T-t path and tectonic evolution of the Archaean rocks in southern Jilin Province. Miner. Petrol. Mag. 13, 23-32 in Chinese.

Geng, Y.S., Wu, J.S., 1990. Geochemistry and evolution of the early Precambrian mafic rocks in Wutai-Taihangshan area. Precambrian Geol. 4, 167-174 in Chinese.

Graham, C.M., Powell, R., 1984. A garnet-hornblende geothermometer: calibration, testing, and application to the Pelona Schist, Southern California. J. Metamorph. Geol. 2, 13-31.

Guo, J.H., Bian, A.G., Shi, X., 1996. High-pressure granulites, retrograded eclogites and granites in the Early Precambrian Sanggan structural belt. In: Zhai, M.G. (Ed.), Granulites and Lower Continental Crust in the North China Craton. Seismological Press, Beijing, pp. 21-54.

Harley, S.L., 1984. The solubility of alumina in orthopyroxene coexisting with garnet in $\mathrm{FeO}-\mathrm{MgO}-\mathrm{Al}_{2} \mathrm{O}_{3}-\mathrm{SiO}_{2}$ and $\mathrm{CaO}-\mathrm{FeO}-\mathrm{MgO}-\mathrm{Al}_{2} \mathrm{O}_{3}-\mathrm{SiO}_{2}$. J. Petrol. 25, 665-696.

Harley, S.L., 1985. Garnet-orthopyroxene bearing granulites from Enderby Land, Antarctica: metamorphic pressuretemperature-time evolution of the Archaean Napier Complex. J. Petrol. 26, 819-856.

Harley, S.L., 1988. Proterozoic granulites from the Rauer Group, East Antarctica, I. Decompressional pressure-temperature paths deduced from mafic and felsic gneisses. J. Petrol. 29, 1059-1095.

Harley, S.L., 1989. The origins of granulites: a metamorphic perspective. Geol. Mag. 126, 215-247.

Heinrich, C.A., 1982. Kyanite-eclogite to amphibolite facies evolution of hydrous mafic and pelitic rocks, Adula Nappe, central Alps. Contrib. Miner. Petrol. 81, 30-38.

Hensen, B.J., Green, D.H., 1972. Experimental study of the stability of cordierite and garnet in pelitic compositions at high pressures and temperatures: II. Compositions without excess alumino-silicate. Contrib. Mineral. Petrol. 35, 191214.

Hodges, K.V., Crowley, P.D., 1985. Error estimation in empirical geothermometry and geobarometry for pelitic system. Am. Miner. 70, 702-709.

Holdaway, M.J., Murkhopadhyay, B.A., 1993. A reevaluation of the stability relations of andalusite: Thennochemical data and phase diagram for the aluminum silicates. Am. Miner. 78, 298-315. 
Holland, T.J.B., Blundy, J., 1994. Non-ideal interactions in calcic amphiboles and their bearing on amphibole-plagioclase thermometry. Contrib. Mineral. Petrol. 116, 433447.

Holland, T.J.B., Powell, R., 1992. Plagioclase feldspar activitycomposition relations based on Darken's Quadratic Formalism and Landau theory. Am. Miner. 77, 53-61.

Holland, T.J.B., Powell, R., 1998. An internally consistent thermodynamic data set for phases of petrological interest. J. Metamorph. Geol. 16, 309-343.

Huang, J.Q., 1977. The basic outline of China tectonics. Acta Geol. Sin. 52, 117-135 in Chinese.

Huang, X., Bai, Z., DePaolo, D.J., 1986. Sm-Nd isotope study of early Archaean rocks, Qianan, Hebei Province, China. Geochim. Cosmochim. Acta 50, 625-631.

Jahn, B.M., Auvray, B., Cornichet, J., Bai, Y.D., Shen, Q.H., Liu, D.Y., 1987. 3.5 Ga old amphibolites from eastern Hebei Province, China: field occurrence, petrography, Sm$\mathrm{Nd}$ isochron age and REE geochemistry. Precambrian Res. 34, 311-346.

Jin, W., Li, S.X., Liu, X.S., 1991. The Metamorphic dynamics of Early Precambrian highgrade metamorphic rocks series in Daqing-Ulashan area, Inner Monglia. Acta Petrol. Sin. 7, 27-35 in Chinese with English abstract.

Kohn, M.J., Spear, F.S., 1990. Two new geobarometers for garnet amphibolites, with applications to southern Vermont. Am. Miner. 75, 89-96.

Koziol, A.M., Newton, R.C., 1988. Redetermination of the anorthite breakdown reaction and improvement of the plagioclase-garnet- $\mathrm{Al}_{2} \mathrm{SiO}_{5}-$ quartz geobarometer. Am. Mineral. 73, 216-223.

Kreze, R., 1983. Symbols for rock-forming minerals. Am. Miner. 68, 277-279.

Kröner, A., Compston, W., Zhang, G.W., Guo, A.L., Cui, W.Y., 1987. Single grain zircon ages for Archean rocks from Henan, Hebei and Inner Mongolia, China, and tectonic implications. International symposium on the tectonic evolution and dynamics of continental lithosphere, Beijing, Abstracts.

Kröner, A., Compston, W., Zhang, G.W., Guo, A.L., Todt, W., 1988. Ages and tectonic setting of Late Archean greenstone-gneiss terrain in Henan Province, China, as revealed by single-grain zircon dating. Geology 16, 211215.

Kröner, A., Cui, W.Y., Wang, W.Y., Wang, C.Q., Nemchin, A.A., 1998. Single zircon ages from high-grade rocks of the Jianping Complex, Liaoning Province, NE China. J. Asian Earth Sci. 16, 519-532.

Li, Z.L., 1993. Metamorphic P-T-t path of the Archaean rocks in the eastern Shandong Province and its implications. Shandong Geol. 9, 31-41 in Chinese.

Li, S.X., Liu, X.S., Zhang, L.Q., 1987. Granite-greenstone belt in Sherteng area, Inner Mongolia. China J. Changchun Univ. Sci. Tech. 17, 81-102 in Chinese.

Li, J.L., Wang, K.Y., Wang, C.Q., Liu, X.H., Zhao, Z.Y., 1990. Early Proterozoic collision orogenic belt in Wutaishan area. China Sci. Geol. Sin. 1, 1-11 in Chinese.
Li, J.H., Zhai, M.G., Li, Y.G., Zhang, Y.G., 1998. Discovery of Early Archean high-pressure granulites in LuanpingChengde area, Northern Hebei, and their tectonic implications. Acta Petrol. Sin. 14, 34-41 in Chinese with English abstract.

Liu, F.L., 1995. Metamorphic mineral-fluid evolution and tectonic environments of the granulite facies terrane in the Huaian-Datong area. Ph.D. dissertation, Changchun University of Science and Technology, Changchun in Chinese with English Abstract.

Liu, S.W., 1996a. P-T path of granulites in the Fuping area. Geol. J. Univ. 2, 75-84.

Liu, Z.H., 1996b. Tectonometamorphic evolution of the Wutai Complex: Constrains on the gold deposits. Ph.D. dissertation, Changchun University of Earth Sciences, Changchun, pp. 1-200 in Chinese with English abstract.

Liu, S.W., Liang, H.H., 1997. Metamorphism of Al-rich gneisses from the Fuping Complex, Taihang Mountain, China. Acta Petrol. Sin. 13, 303-312 in Chinese.

Liu, D.Y., Page, R.W., Compston, W., Wu, J.S., 1985. U-Pb zircon geochronology of Late Archean metamorphic rocks in the Taihangshan-Wutaishan area, North China. Precambrian Res. 27, 85-109.

Liu, D.Y., Nutman, A.P., Compston, W., Wu, J.S., Shen, Q.H., 1992. Remnants of 3800 crust in the Chinese Part of the Sino-Korean craton. Geology 20, 339-342.

Liu, X.S., Jin, W., Li, S.X., Xu, X.C., 1993. Two types of Precambrian high-grade metamorphism, Inner Mongolia, China. J. Metamorph. Geol. 11, 499-510.

Lu, L.Z., 1991. Metamorphic P-T-t path of the Archean granulite-facies terrains in Jining area, Inner Mongolia and its tectonic implications. Acta Petrol. Sin. 8, 1-12 in Chinese.

Lu, L.Z., Jin, S.Q., 1993. P-T-t paths and tectonic history of an early Precambrian granulite facies terrane, Jining district, southeastern Inner Mongolia, China. J. Metamorph. Geol. 11, 483-498.

Lu, L.Z., Jin, S.Q., Xue, X.C., Liu, F.L., 1992. Petrogenesis of Early Precambrian Khondalite Series in Southern Inner Mongolia and its Potential Mineral Resources. Jilin Press of Science and Technology, Changchun, pp. 107-130 in Chinese with English abstract.

Mäder, U.K., Percial, J.A., Berman, R.G., 1994. Thermobarometry of garnet-clinopyroxene-homblende granulite from the Kapuskasing Structural Zone. Can. J. Earth Sci. 31, 1134-1145.

Mei, H.L., 1994. P-T-t path and tectonic evolution of Paleoproterozoic metamorphic rocks in Zhongtiaoshan area. Geol. Rev. 40, 36-45 in Chinese with English abstract.

Mengel, F., Rivers, T., 1991. Decompression reactions and $\mathrm{P}-\mathrm{T}$ conditions in high-grade rocks, Northern Labrador: $\mathrm{P}-\mathrm{T}-\mathrm{t}$ paths from individual samples and implications for Early Proterozoic tectonic evolution. J. Petrol. 32, 139167.

Mezger, K., Bohlen, S.R., Hanson, G.N., 1990. Metamorphic history of the Archean Pikwitonei Granulite Domain and the Cross Lake Subprovince, Superior Province, Manitoba. Can. J. Petrol. 31, 483-517. 
Möller, C., 1998. Decompressed eclogites in the Sveconorwegian (-Grenvillian) orogen of SW Sweden: petrology and tectonic implications. J. Metamorph. Geol. 16, 641-656.

McMullin, D., Berman, R.G., Greenwood, H.J., 1991. Calibration of the SGAM thermobarometer for pelitic rocks using data from equilibrium experiments and natural assemblages. Can. Miner. 29, 889-908.

Newton, R.C., Perkins, D., 1982. Thermodynamic calibration of geobarometers based the assemblage garnet-plagioclase-orthopyroxene (clinopyroxene)-quartz. Am. Mineral. 67, 203-222.

Paria, P., Bhattacharya, A., Sen, A., 1988. The reaction garnet + clinopyroxene + quartz $=2$ orthopyroxene + anorthite: a potential geobarometry for granulites. Contrib. Mineral. Petrol. 99, 126-133.

Plyusnina, L.P., 1982. Geothermometry and geobarometry of plagioclase-hornblende bearing assemblages. Contrib. Mineral. Petrol. 80, 140-146.

Powell, R., Holland, T.J.B., 1994. Optimal geothermometry and geobaromemetry. Am. Miner. 79, 120-133.

Powell, R., Holland, T.J.B., Worley, B., 1998. Calculated phase diagrams involving solid solutions via non-linear equations, with examples using THERMOCALC. J. Metamorph. Geol. 16, 577-588.

Qiao, X.F., 1985. Geochronology of the Xiyanghe Group in southern Shanxi Province and its implications. Acta Geol. Sin. 59, 258-269 in Chinese with English abstract.

Qiao, G.S., Wang, K.Y., Guo, Q.F., Zhang, G.C., 1987. $\mathrm{Sm}-\mathrm{Nd}$ isotopic dating of the Paleoarchean rocks in the eastern Hebei Province. Geol. Sci. 2, 158-165 in Chinese.

Ren, J.S., 1980. Tectonics and Evolution of China. Science Press, Beijing, pp. 64-75.

Rubie, D.C., 1990. Role of kinetics in the formation and preservation of eclogites. In: Carswell, D.A. (Ed.), Eclogite Facies Rocks. Blackie, Glasgow, pp. 111-140.

Sandiford, M., Powell, R., 1986. Deep crustal metamorphism during continental extension: ancient and modern examples. Earth Planet. Sci. Lett. 79, 151-158.

Selverstone, J., Chamberlain, C.P., 1990. Apparent isobaric cooling paths from granulites: two counterexamples from British Colombia and New Hampshire. Geology 18, 307310.

Shen, Q.H., Liu, D.Y., Wang, P., Gao, J.F., Zhang, Y.F., 1987. $\mathrm{U}-\mathrm{Pb}$ and $\mathrm{Rb}-\mathrm{Sr}$ isotopic ages of metamorphic rock series from the Jining Group, southern Inner Mongolia. J. China Inst. Geol. 16, 165-178 in Chinese.

Shen, Q.H., Zhang, Y.F., Gao, J.F., Wang, P., 1990. Archean metamorphic rocks in the central and southern parts of Inner Mongolia. Chin. Acad. Geol. Sci. Bull. 21, 1-192 in Chinese with English abstract.

Shen, Q.H., Zhang, Z.Q., Geng, Y.S., Tang, S.H., 1994. Petrology, geochemistry and isotopic ages of the garnetbearing basic metamorphic rocks from the Dadonggou area, northwestern Hebei Province, China. In: Qian, X.L., Wang, R.M. (Eds.), Geological Evolution of Granulite Terranes in the North China Craton. Seismological Press, Beijing, pp. 120-129 in Chinese.
Smelov, A.P., Beryozkin, V.I., 1993. Retrograded eclogites in the Olekma granite-greenstone region, Aldan Shield, Siberia. Precambrian Res. 62, 419-430.

Song, B., Nutman, A.P., Liu, D.Y., Wu, J.S., 1996. 3800 to 2500 Ma crustal evolution in Anshan area of Liaoning Province, Northeastern China. Precambrian Res. 78, 7994.

Spear, F.S., 1989. Petrological determination of metamorphic pressure-temperature-time paths. In: Spear, F.S., Peacock, S.M. (Eds.), 'Metamorphic Pressure-TemperatureTime Paths', American Geophysical Union Short Corse in Geology 7, pp. 1-55.

Sun, M., Armstrong, R.L., Lambert, R.S.t.J., 1992. Petrochemistry and $\mathrm{Sr}, \mathrm{Pb}$ and $\mathrm{Nd}$ isotopic geochemistry of Early Precambrian rocks, Wutaishan and Taihangshan areas, China. Precambrian Res. 56, 1-31.

Sun, D.Y., Liu, Z.H., Zheng, C.Q., 1993a. Metamorphism and Tectonic Evolution of Early Precambrian Rocks in Fushun area, the northern Liaoning Province. Seismol. Press, Beijing, pp. 90-120 in Chinese.

Sun, D.Z., Hu, W.X., Tang, M., Zhao, F.Q., 1993b. The Geochronological Framework and Crustal Structures of Precambrian Basement in the Zhongtiaoshan Area. Geological Publishing House, Beijing, pp. 1-180 in Chinese.

Thompson, A.B., 1976. Mineral reactions in pelitic rocks: II. Calculations of some $\mathrm{P}-\mathrm{T}-\mathrm{X}(\mathrm{Mg}-\mathrm{Fe})$ phase relation. Am. J. Sci. 276, 425-454.

Thompson, A.B., England, P.C., 1984. Pressure-temperaturetime paths of regional metamorphism, II. Their influences and interpretation using mineral assemblages in metamorphic rocks. J. Petrol. 25, 929-955.

Tian, Y.Q., 1991. Geology and Mineralisation of the WutaiHengshan Greenstone Belt. Shanxi Science and Technology Press, Taiyuan, pp. 137-152 in Chinese.

Tian, Y.Q., Liang, Y.F., Fan, S.K., Zhu, B.Q., Chen, L.W., 1992. Geochronology and $\mathrm{Nd}$ isotopic evolution of the Hengshan Complex. Geochimica 3, 255-263 in Chinese.

Vielzeuf, D., 1983. The spinel and quartz associations in high grade xenolithos from Tallante (SE Spain) and their potential use in geothermometry and barometry. Contrib. Mineral. Petrol. 82, 301-311.

Wang, R.M., Chen, Z.Z., Chen, F., 1991. Grey gneisses and high-pressure granulites enclaves in Hengshan area, Shanxi Province, and their geological significance. Acta Petrol. Sin. 7, 36-45.

Wang, R.M., Lai, X.Y., Dong, W.D., Ma, J., Tang, B., 1994. Some evidence for the late Archean collisional belt in the western Hebei Province. In: Qian, X.L., Wang, R. (Eds.), Geological Evolution of the Granulite Terrains in the Part of the North China Craton. Seismological Press, Beijing, pp. 7-20.

Wang, J., Lu, S.N., Li, H.M., Wang, R.Z., Sun, Y.F., Li, H.K., Li, S.Q., 1995. Geochronological framework of metamorphic rocks in the middle part of Inner Mongolia. Tianjin Inst. Geol. Miner. Res. Bull. 29, 1-76 in Chinese. 
Wang, K.Y., Li, J.L., Hao, J., Li, J.H., Zhou, S.P., 1996. The Wutaishan mountain belt within the Shanxi Province, Northern China: a record of late Archean collision tectonics. Precambrian Res. 78, 95-103.

Wang, R.Z., Yan, Y.Y., Li, H.M., Lin, Y.X., 1997. The early Precambrian geochronological framework in the Wutaishan area. Shanxi Progr. Precambrian Res. 20, 44-50.

Wang, K.Y., Li, J.L., Hao, J., Li, J.H., Zhou, S.P., 1997 a. Late Archean mafic-ultramafic rocks from the Wuatishan, Shanxi Province: a possible ophiolite melange. Acta Petrol. Sin. 13, 139-151 in Chinese.

Wang, K.Y., Hao, J., Zhou, S.P., Wilde, S.A., Cawood, P.A., 1997b. Orogenic events in the Wutai area: constraints from SHRIMP zircon dating. Chin. Sci. Bull. 42, 1295-1298.

Wang, K.Y., Hao, J., Cawood, P., Wilde, S.A., 1997c. Highpressure metamorphism in kyanite-bearing schists from the original Jingangku, Formation of the Wutaishan. Proceedings of the 30th IGC. Precambrian Geol. Metamorph. Petrol. 17, 213-220.

Wells, P.R.A., 1980. Thermal models for magmatic accretion and subsequent metamorphism of continental crust. Earth Planet. Sci. Lett. 46, 253-265.

Wilde, S.A., Cawood, P., Wang, K.Y., 1997. The relationship and timing of granitoid evolution with respect to felsic volcanism in the Wutai Complex, North China Craton. Proceedings of the 30th IGC. Precambrian Geol. Metamorph. Petrol. 17, 75-88.

Wilde, S.A., Cawood, P.A., Wang, K.Y., 1998. SHRIMP $\mathrm{U}-\mathrm{Pb}$ data of granites and gneisses in the TaihangshanWutaishan area: implications for the timing of crustal growth in the North China Craton. China Sci. Bull. 43, 144.

Wu, C.H., Zhong, C.T., 1998. The Paleoproterozoic SW-NE collision model for the central North China Craton. Progr. Precambrian Res. 21, 28-50 in Chinese.

Wu, C.H., Zhong, C.T., Chen, Q.A., 1997. Ages of khondalites in the Jin-Ji-Meng high-grade terrains, North China Craton. Acta Petrol. Sin. 13, 289-302 in Chinese.

Wu, J.S., Geng, Y.S., Shen, Q.H., Liu, D.Y., Li, Z.L., Zhao, D.M., 1991. The Early Precambrian Significant Geological Events in the North China Craton. Geological Publishing House, Beijing, pp. 1-115 in Chinese with English abstract.

Xu, R.H., Zhu, M., Chen, F.K., Guo, J.H., 1995. The timing of the Longquanguan ductile shear zone. Quat. Sci. 4, $332-342$.
Yardley, B.W.D., 1989. An Introduction to Metamorphic Petrology. Longman Group UK, Harlow, pp. 49-51.

Zhai, M.G., Guo, J.H., Yan, Y.H., 1992. Discovery and preliminary study of the Archean high-pressure granulites in the North China. Chin. Sci. 12B, 1325-1330.

Zhai, M.G., Guo, J.H., Li, Y.G., Yan, Y.H., 1995. Discovery of Archean retrograded eclogites in the North China Craton and their tectonic implications. Chin. Sci. Bull. 40, $1590-1594$.

Zhang, Q.C., 1988. Sm-Nd isotopic ages of the upper sequence of the Wutai Group in the Lüliang area. J. Chengdu Univ. Geol. 15, 76-85 in Chinese.

Zhang, C.H., 1990. Metamorphism of Early Precambrian Rocks in the Eastern Hebei Province. Geological Publishing House, Beijing, p. 212 in Chinese.

Zhang, G.W., Bai, Y.B., Sun, Y., Guo, A.L., Zhou, D.W., Li, T.H., 1985. Composition and evolution of the Archean crust in central Henan, China. Precambrian Res. 27, 7-35.

Zhang, Z.Q., Wu, J.S., Yie, X.J., 1991. REE, Rb-Sr and $\mathrm{Sm}-\mathrm{Nd}$ ages of the Archaean metamorphic rocks of the Lower Fuping Subgroup and their implications. Sin. Geochim. 2, 118-126 in Chinese with English abstract.

Zliang, J.S., Dirks, H.G.M., Passchier, C.W., 1994. Extensional collapse and uplift in a polymetamorphic granulite terrain in the Archean and Paleoproterozoic of north China. Precambrian Res. 67, 37-57.

Zhao, G.C., Wilde, S.A., Cawood, P.A., Lu, L.Z., 1998. Thermal evolution of Archean basement rocks from the eastern part of the North China Craton and its bearing on tectonic setting. Inter. Geol. Rev. 40, 706-721.

Zhao, G.C., Wilde, S.A., Cawood, P.A., Lu, L.Z., 1999a. Tectonothermal history of the basement rocks in the western zone of the North China Craton and its tectonic implications. Tectonophysics 310, 223-240.

Zhao, G.C., Cawood, P.A., Lu, L.Z., 1999b. Petrology and $\mathrm{P}-\mathrm{T}$ history of the Wutai amphibolites: implications for tectonic evolution of the Wutai Complex, China. Precambrian Res. 93, 181-199.

Zhao, G.C., Cawood, P.A., Wilde, S.A., 1999c. Polymetamorphism of Archean mafic granulties from the Trans-North China Orogen: textural evidence and tectonic implications. Austr. Geol. Soc. Abstr. 54, 113-114.

Zhao, G.C., Wilde, S.A., Cawood, P.A., Lu, L.Z., in press. Petrology and $\mathrm{P}-\mathrm{T}$ path of the Fuping mafic granulites: Implications for tectonic evolution of the central zone of the North China Craton. J. Metamorph. Geol. 18. 